\author{
LHCb upgrade: \\ Plans and physics potential \\ U. Marconi \\ INFN Bologna (Italy) \\ On behalf of the LHCb Collaboration
}




\section{LHCb upgrade}

- The upgrade of the LHCb detector and DAQ system aims to enable the collaboration to run the experiment at a luminosities 5 times greater than presently:

$$
\mathrm{L}_{1}=4 . \times 10^{32} \mathrm{~cm}^{-2} \mathrm{~s}^{-1} \rightarrow \mathrm{L}_{2}=2 . \times 10^{33} \mathrm{~cm}^{-2} \mathrm{~s}^{-1}
$$

- Goals:

- Double the present yield: collect $5 \mathrm{fb}^{-1} / \mathrm{y}$ in run 3 and run 4

- Improve considerably the trigger efficiency on hadronic channels $\left(B_{s} \rightarrow \phi \phi\right)$ and on rare decays.

- Expand the scope to the lepton flavor sector, electroweak physics, QCD and exotics searches.

- The upgrade (phase I) will take place during the LS2, foreseen in the years $2019-2020$. 


\section{Single arm forward spectrometer}

Covering about $4 \%$ of the solid angle the detector captures $40 \%$ of the beauty and charm cross-sections.

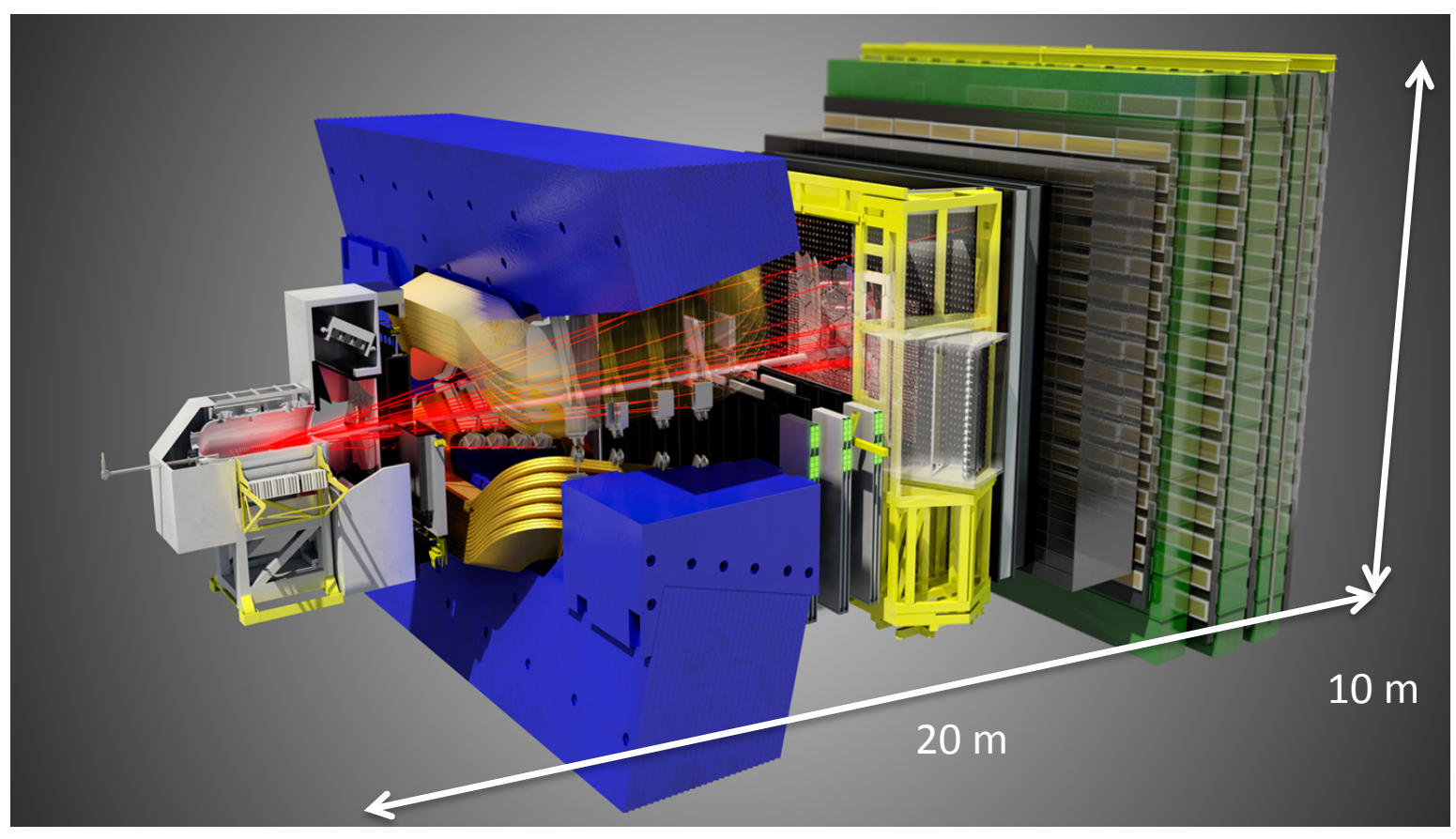

Acceptance $2<\eta<5$

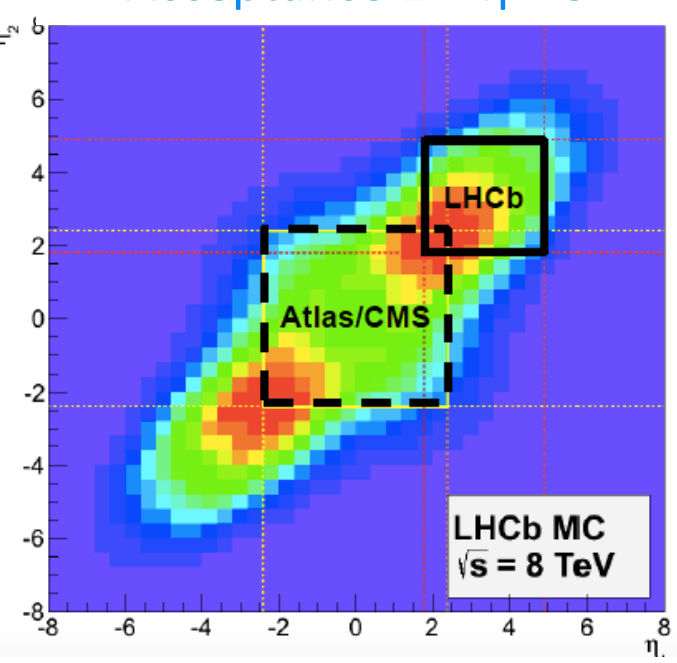

differential cross-section

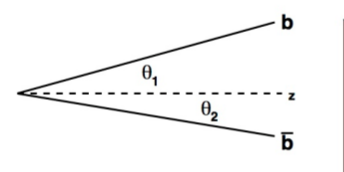

- LHCb measured $\sigma_{\text {beauty }}=75 \mu \mathrm{b}$ at $\mathrm{Vs}=7 \mathrm{TeV}$ in acceptance.

- Charm cross section is 20 times more.

- Cross sections scale linearly with Vs.

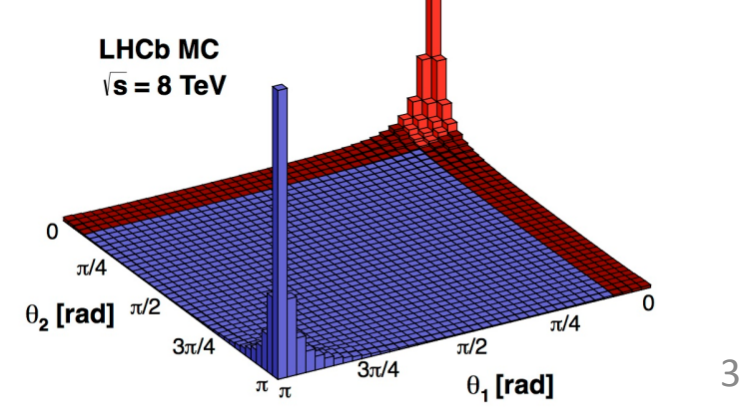




\section{Present detector performance}

- Decay time resolution $\Delta \mathrm{t}: \mathbf{3 0 - 5 0} \mathrm{fs}$

- $\Delta p / p=0.5-1.0 \%$ in the range $5-200 \mathrm{GeV} / \mathrm{c}$

- Muon ID : $\varepsilon(\mu \mid \mu)=95 \%, \varepsilon(\pi \mid \mu) \sim 1 \%$

- RICH ID : $\varepsilon(\mathrm{K} \mid \mathrm{K})=95 \%, \varepsilon(\mathrm{K} \mid \pi) \sim 5 \%$

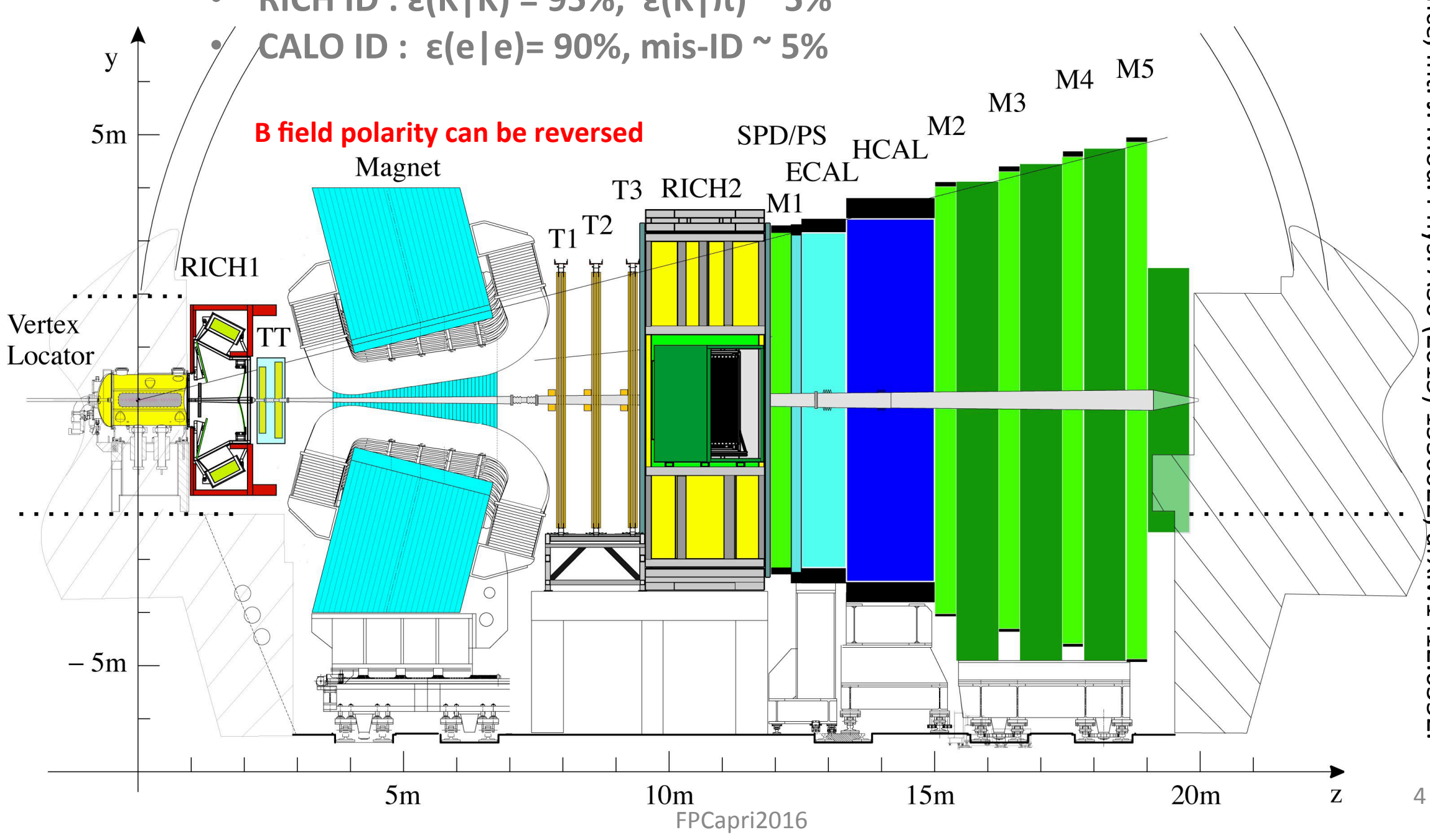




\section{Luminosity}

- Constant luminosity: stable running conditions and selection criteria

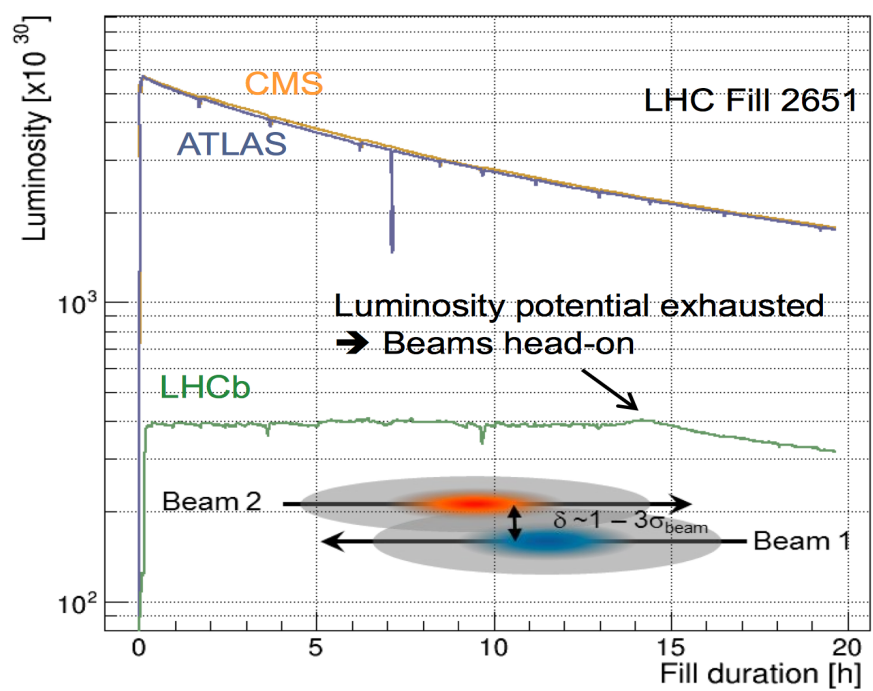

monitoring

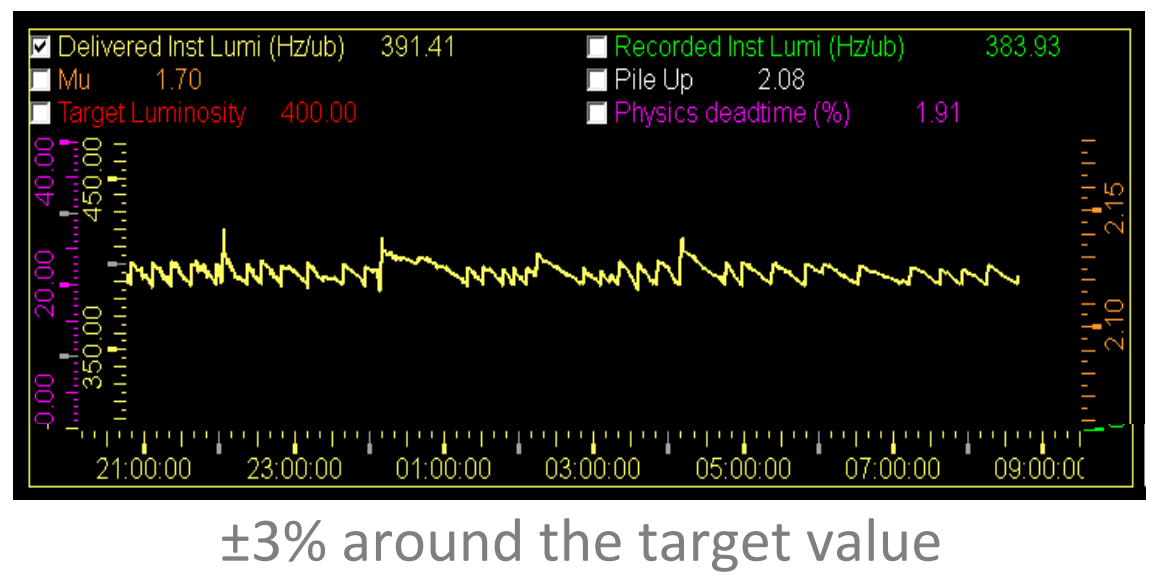

- LHCb was designed to operate with a single collision per bunch crossing, running at $2 . \times 10^{32} \mathrm{~cm}^{-2} \mathrm{~s}^{-1}$, with $25 \mathrm{~ns}$ bunch spacing and 2700 circulating bunches.

- We run at 4. $\times 10^{32} \mathrm{~cm}^{-2} \mathrm{~s}^{-1}$, with 1262 colliding bunches, and 50 ns time spacing: 4 times more collisions per crossing than planned in the detector design. 


\section{Trigger evolution}

\section{$40 \mathrm{MHz}$ bunch crossing rate}
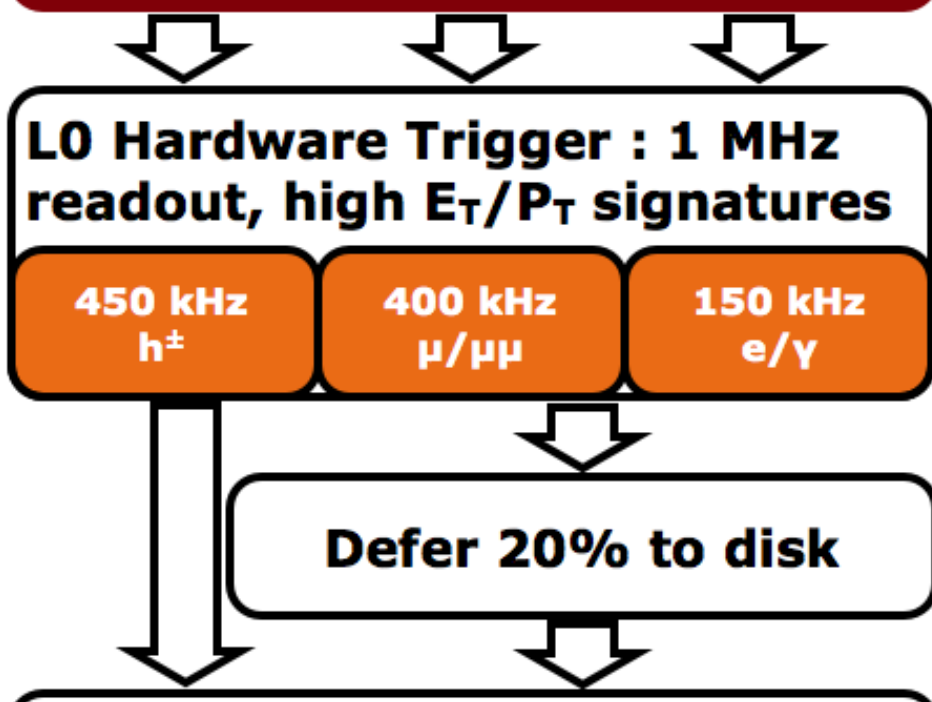

Software High Level Trigger 29000 Logical CPU cores

Offline reconstruction tuned to trigger time constraints

Mixture of exclusive and inclusive selection algorithms
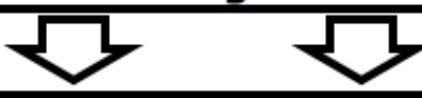

$5 \mathrm{kHz}$ Rate to storage
LHCb 2015 Trigger Diagram

$40 \mathrm{MHz}$ bunch crossing rate
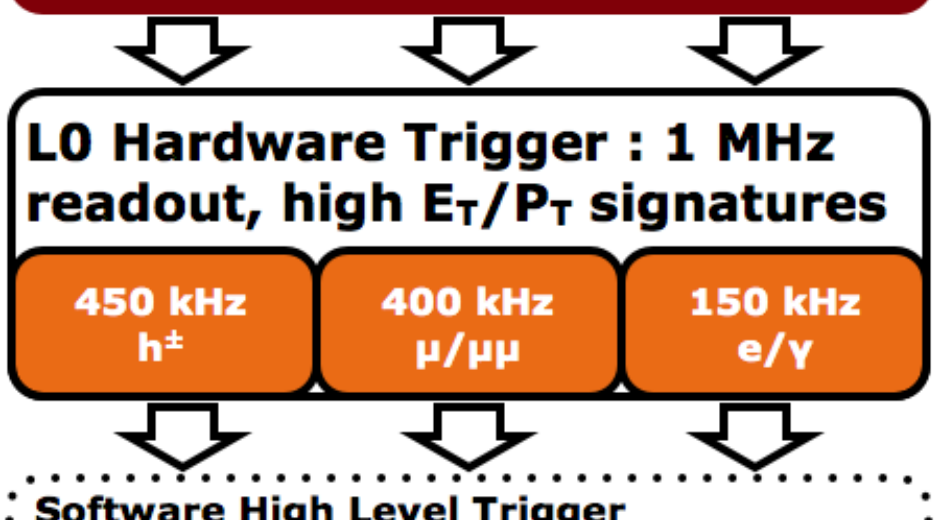

:Software High Level Trigger

Partial event reconstruction, select displaced tracks/vertices and dimuons

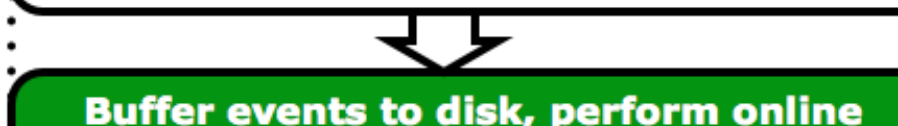

Buffer events to disk, perform online detector calibration and alignment

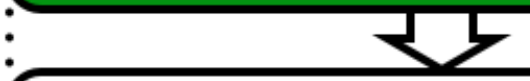

Full offline-like event selection, mixture of inclusive and exclusive triggers

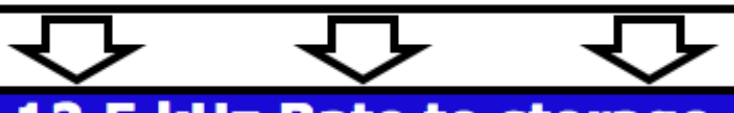

$12.5 \mathrm{kHz}$ Rate to storage 


\section{The $1 \mathrm{MHz}$ readout rate limitation}
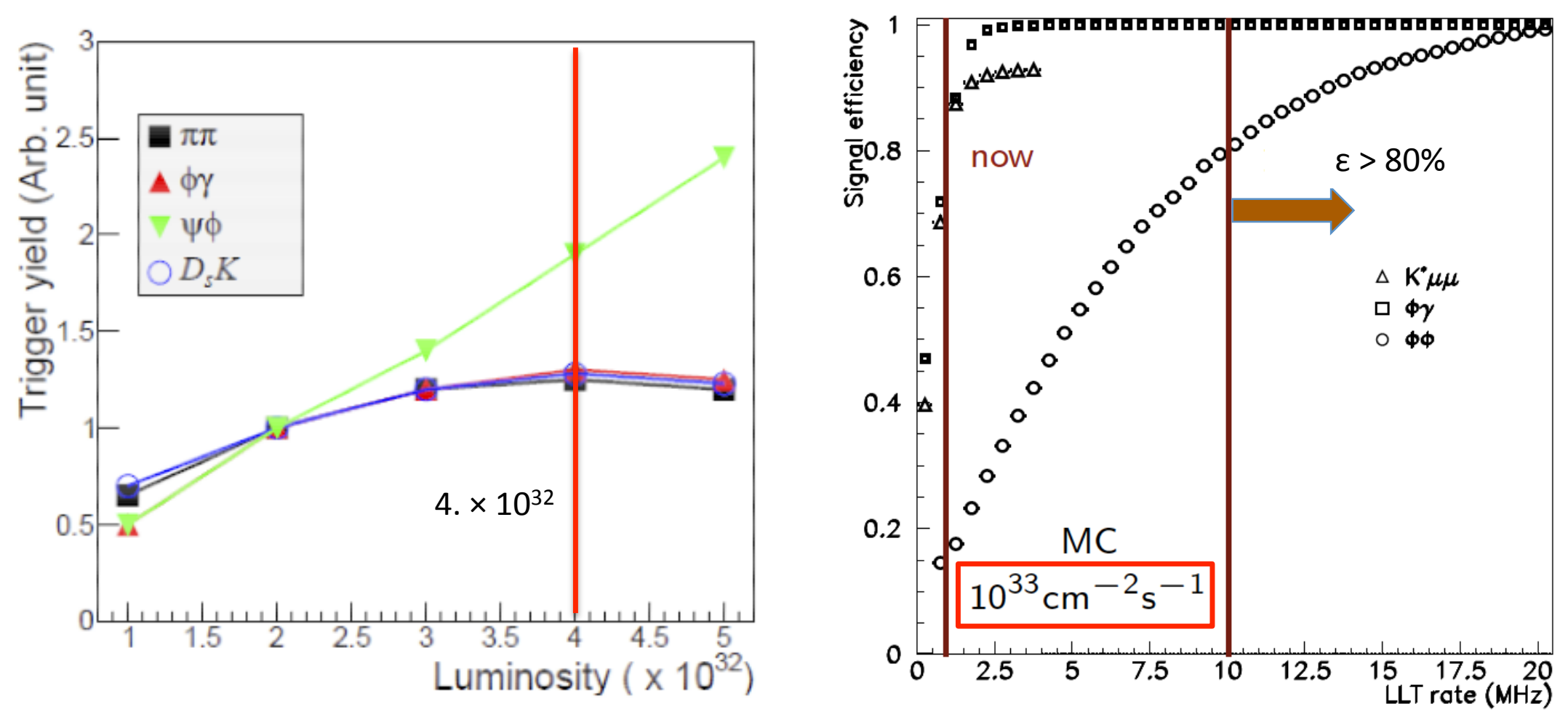

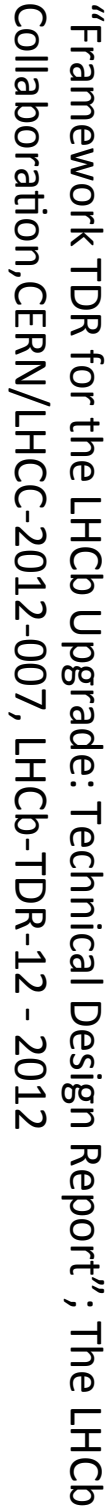




\section{Upgrade main concepts}

\section{Requirements}

- Readout the whole detector at $40 \mathrm{MHz}$.

- Event selection performed by means of the HLT software trigger only.

- Luminosity of $2 . \times 10^{33} \mathrm{~cm}^{-2} \mathrm{~s}^{-1}$

- pp interaction rate: $27 \mathrm{MHz}$

- Average visible interactions: $\mu=5.2$

Poisson mean: $v=7.6$
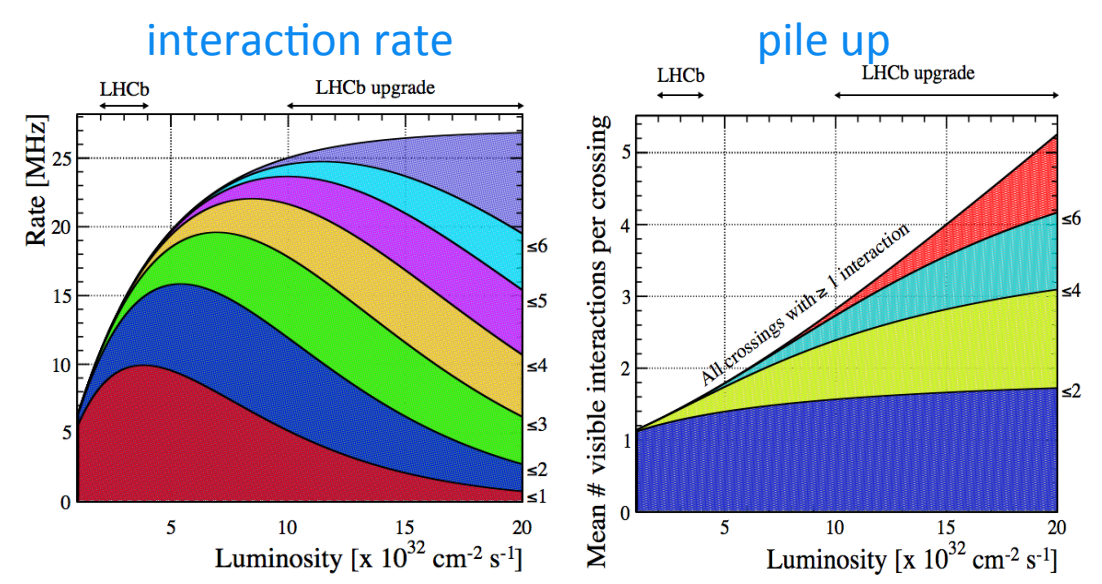

Consequences

- The detector front-end electronics has to be entirely rebuilt because of the new readout requirement.

- New HLT farm and network new LAN technologies and need of powerful many-core processors.

- Rebuild the trackers finer detector granularity to reduce channel's occupancy.

- Consolidate sub-detectors Let them stand the foreseen higher luminosity. 


\section{DAQ Upgrade: $40 \mathrm{MHz}$ PCle based readout}

$5 \mathrm{~Gb} / \mathrm{s}, 300 \mathrm{~m}$ long fibres

Data from the FEE flow

directly to the Event Builder

8800

Event Builder

Versatile Link
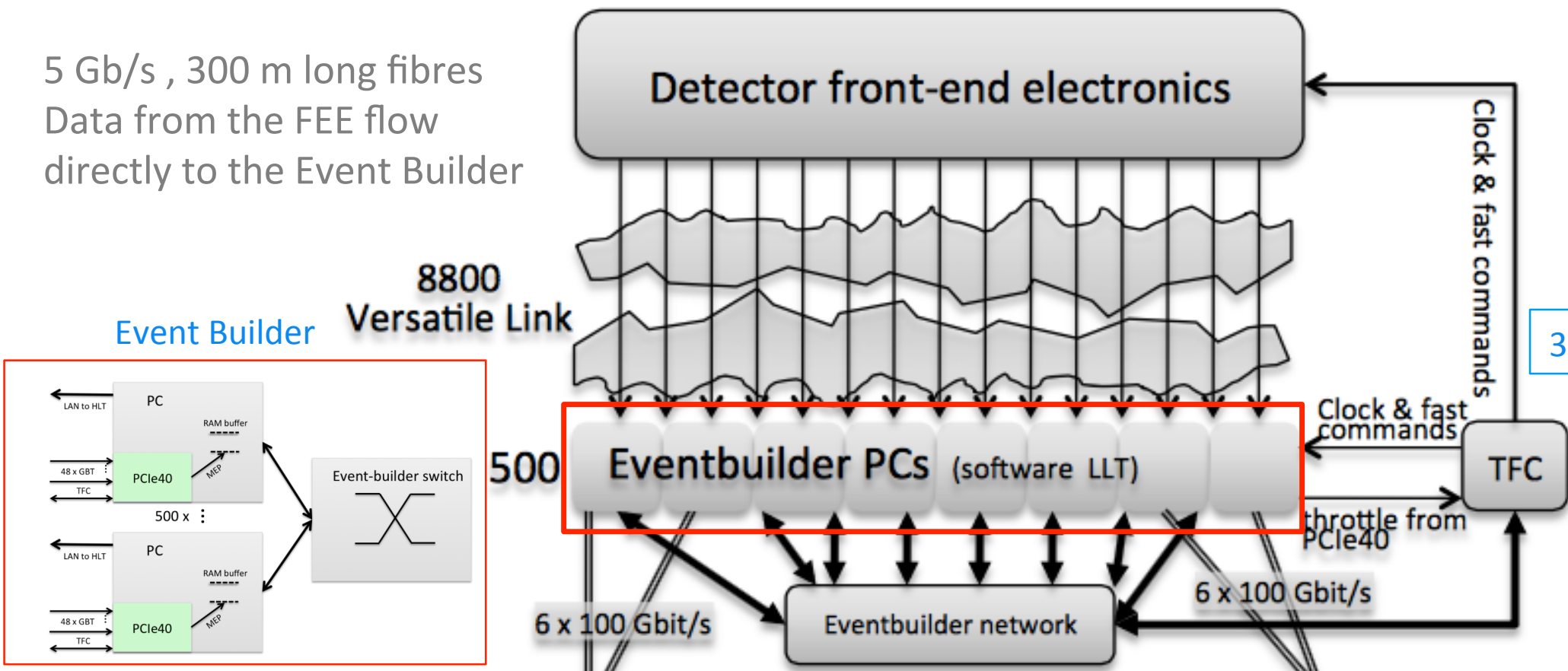

$30 \mathrm{MHz} \times 100 \mathrm{kB} / \mathrm{evt}$

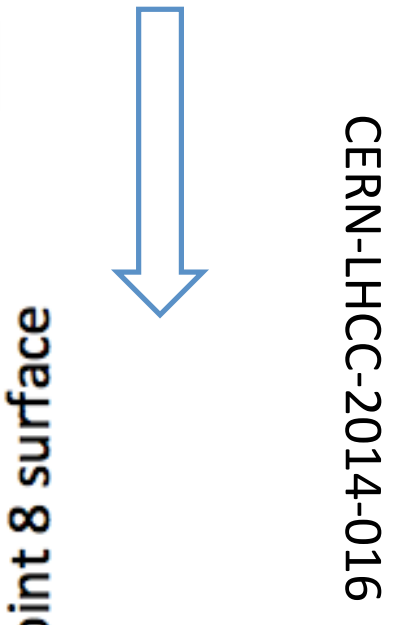

HLT threads

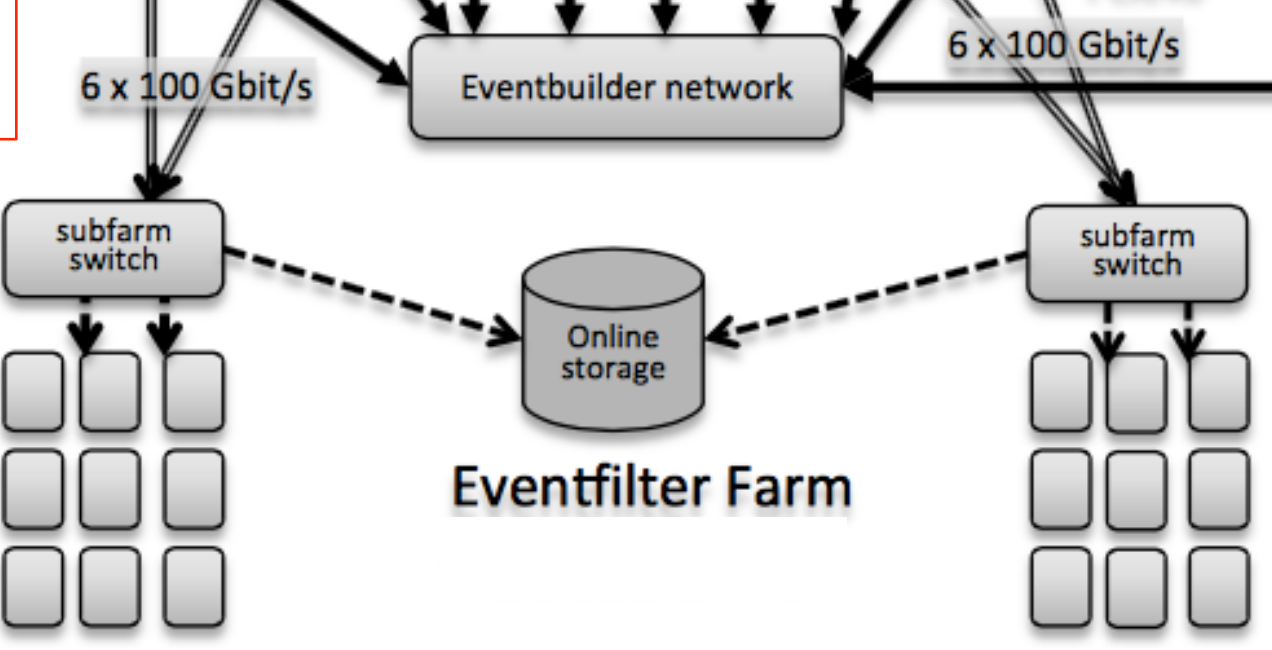

$n \sim 30 \mathrm{MHz} \times 20 \mathrm{~ms} \sim 6 \times 10^{5}$ 


\section{HLT output rate}

Effective input rate: $30 \mathrm{MHz}$, event size $=100 \mathrm{kB}$

\begin{tabular}{|c|c|c|c|c|}
\hline MB events & $b$-hadrons & $c$-hadrons & light, long-lived hadrons & s which had \\
\hline Reconstructed yield & $0.032 \pm 0.001$ & $0.118 \pm 0.001$ & $0.406 \pm 0.002$ & Id he \\
\hline$\epsilon\left(p_{\mathrm{T}}>2 \mathrm{GeV} / c\right)$ & $85.6 \pm 0.6 \%$ & $51.8 \pm 0.5 \%$ & $2.3 \pm 0.1 \%$ & $\begin{array}{l}\text { Which a vert } \\
\text { produced. }\end{array}$ \\
\hline$\epsilon(\tau>0.2 \mathrm{ps})$ & $88.1 \pm 0.6 \%$ & $63.1 \pm 0.5 \%$ & $99.5 \pm 0.1 \%$ & \\
\hline$\epsilon\left(p_{\mathrm{T}}\right) \times \epsilon(\tau) \times \epsilon(\mathrm{LHCb})$ & $27.9 \pm 0.3 \%$ & $22.6 \pm 0.3 \%$ & $2.2 \pm 0.1 \%$ & \\
\hline Output rate & $27 \mathrm{~GB} \mathrm{~s}^{-1}$ & $80 \mathrm{~GB} \mathrm{~s}^{-1}$ & $26 \mathrm{~GB} \mathrm{~s}^{-1}$ & Challenge \\
\hline
\end{tabular}

Rates as a function of decay time cut for part. reco. candidates

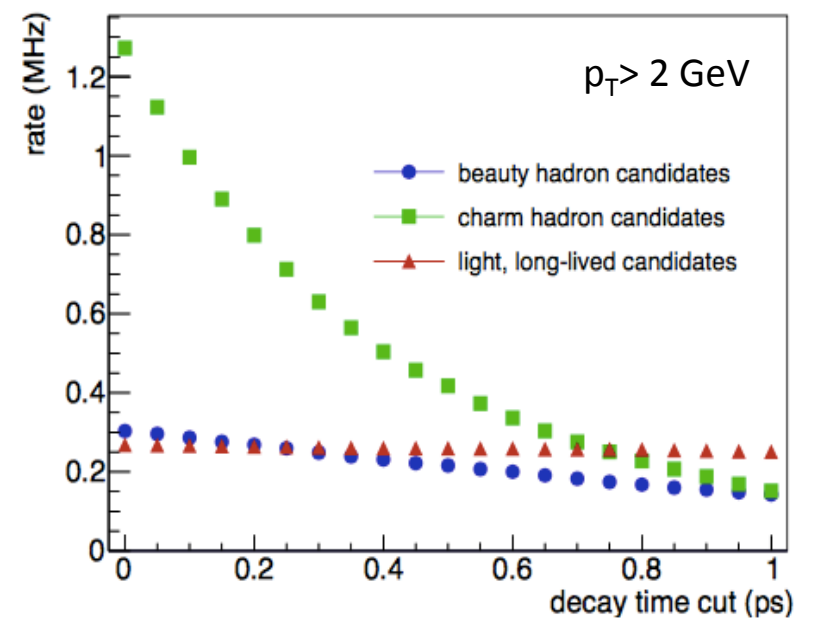

Rates as a function of pT cut for part. reco. candidates

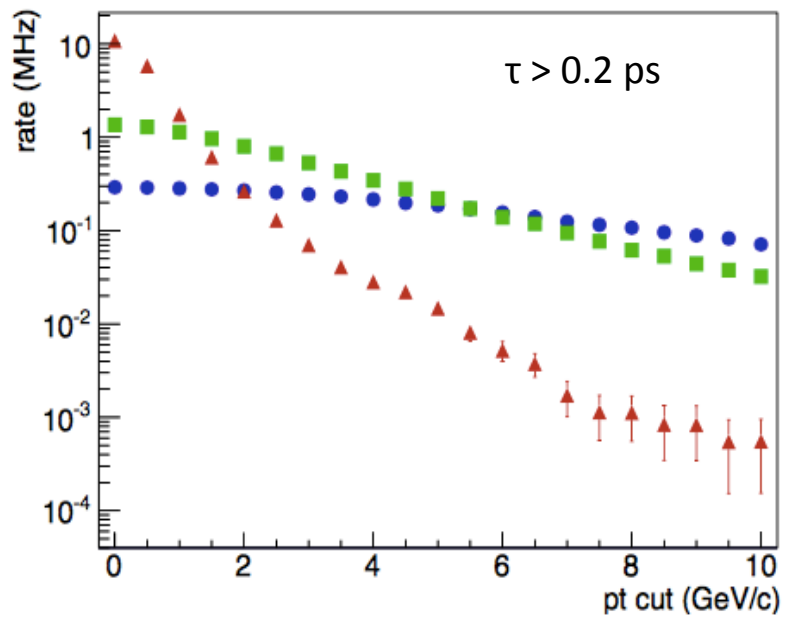

How to limit the HLT output rate? downscale signals, reduce the event size. 


\section{Upgrade of the tracking system}

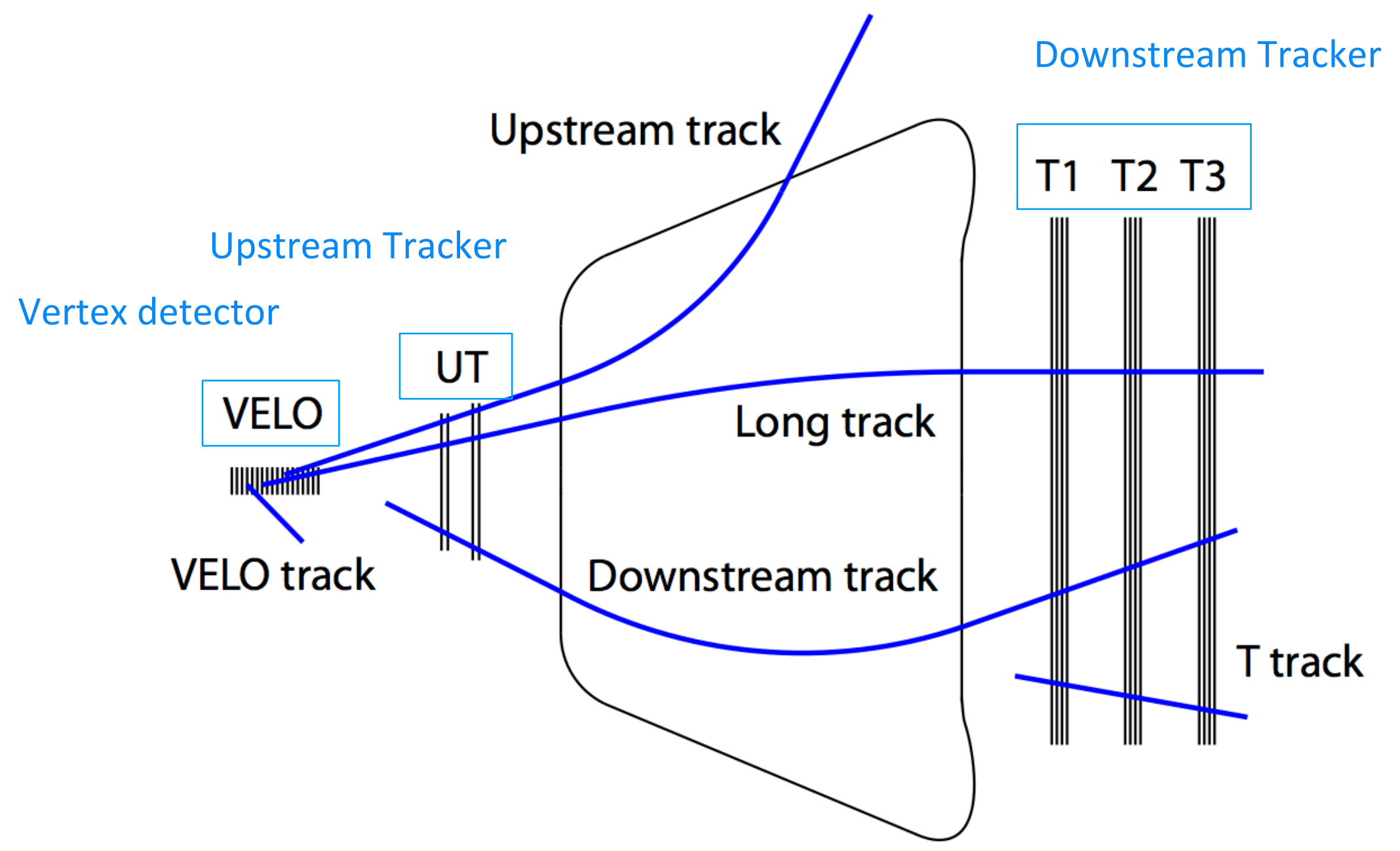




\section{VELO upgrade}

- Silicon pixels for higher granularity and improved resolution

- Pixels of surface area $55 \times 55 \mu \mathrm{m}^{2}$

- Reduced material budget:

- Sensor thickness: $300 \mu \mathrm{m} \rightarrow 200 \mu \mathrm{m}$

- Aluminum foil: $300 \mu \mathrm{m} \rightarrow \leq 250 \mu \mathrm{m}$

- Enlarged acceptance:

- Edge of detector closer to beam

$8.2 \mathrm{~mm} \rightarrow 5.1 \mathrm{~mm}$

- $26 \times 2$ modules, in two retractable halves.

- New readout chip VeloPix with

CMOS $130 \mathrm{~nm}$ technology

- Sustain 400 MRad

- Close to beam $\sim 10^{16} \mathrm{n}_{\text {eq }} \mathrm{cm}^{-2}$ for $50 \mathrm{fb}^{-1}$

- Cooling

- Cool to $-10^{\circ} \mathrm{C}$ to $-15^{\circ} \mathrm{C}$ to prevent thermal runaway, by using micro-channel $\mathrm{CO}_{2}$ cooling

One of the retractable halves

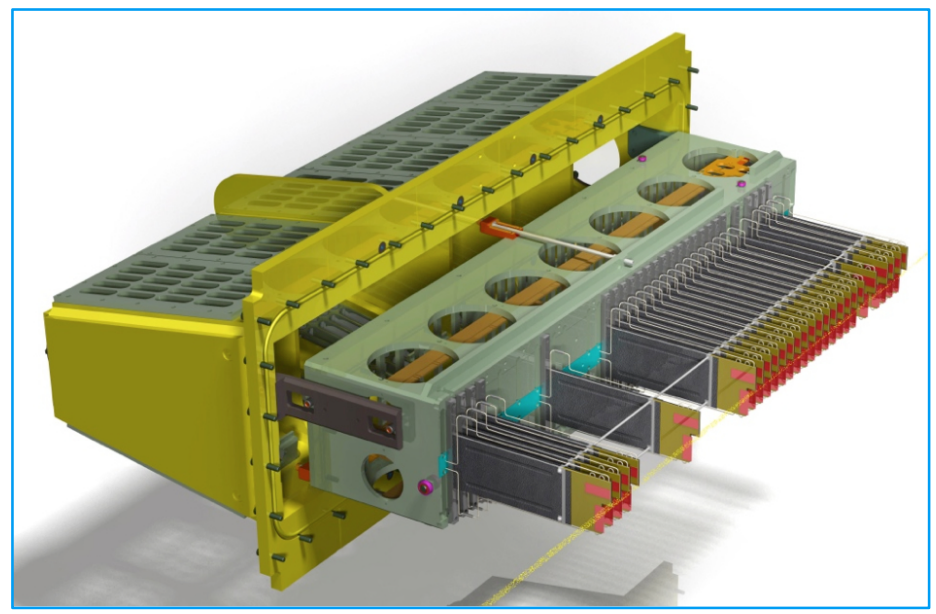

VELO module

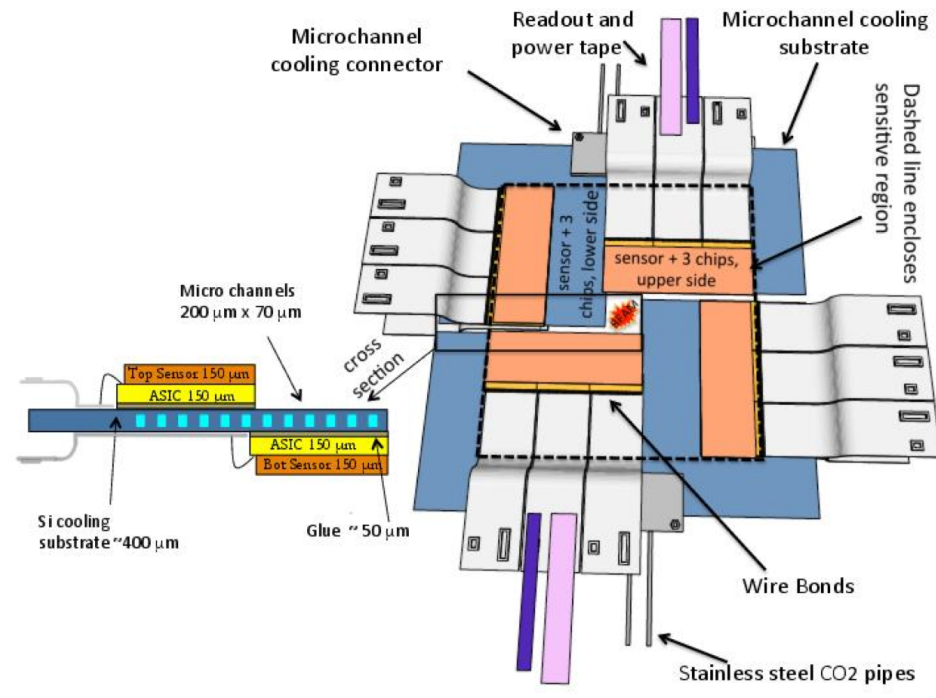




\section{Velo Upgrade (II)}

\section{Velo halves in running position}

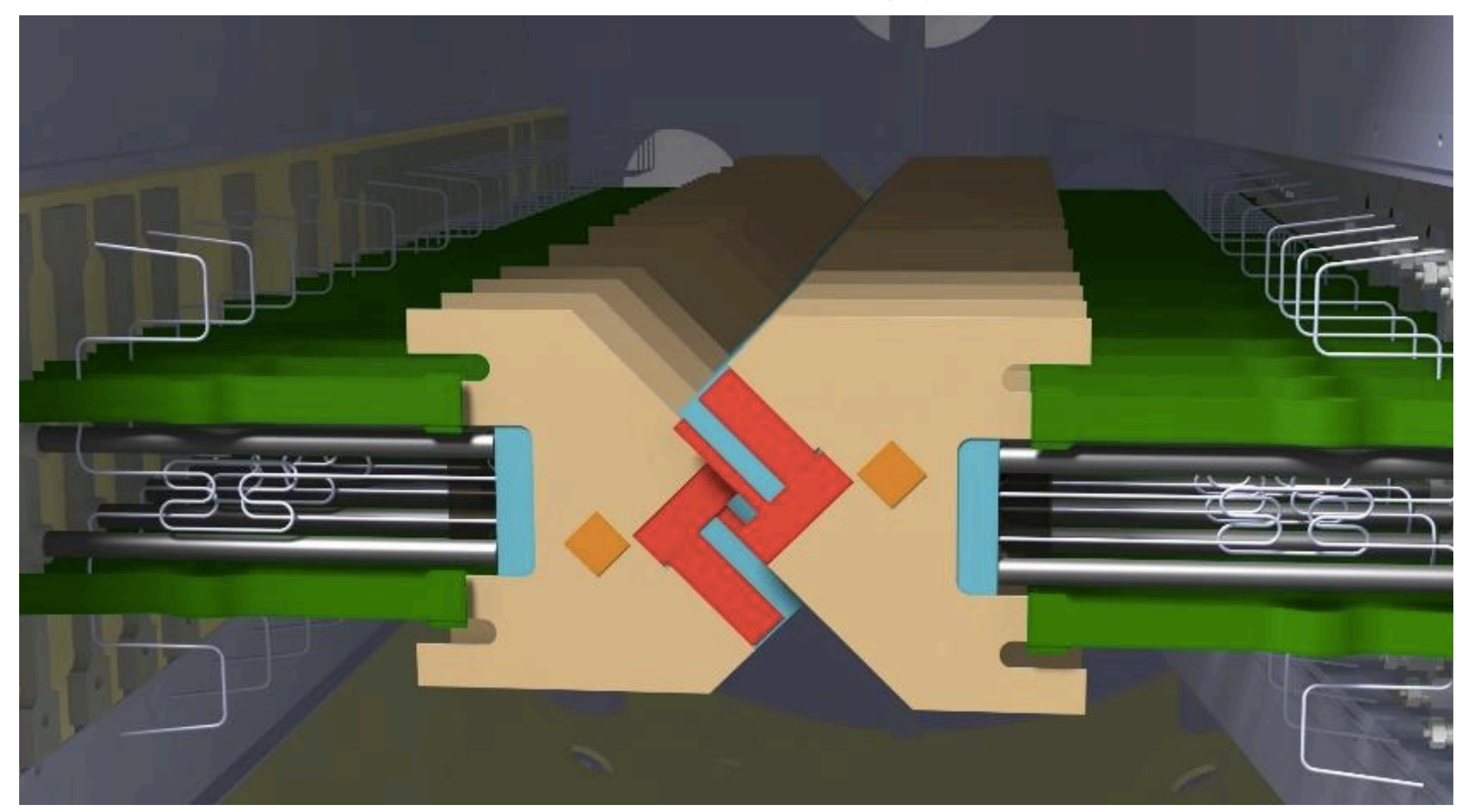




\section{VELO upgrade performance}
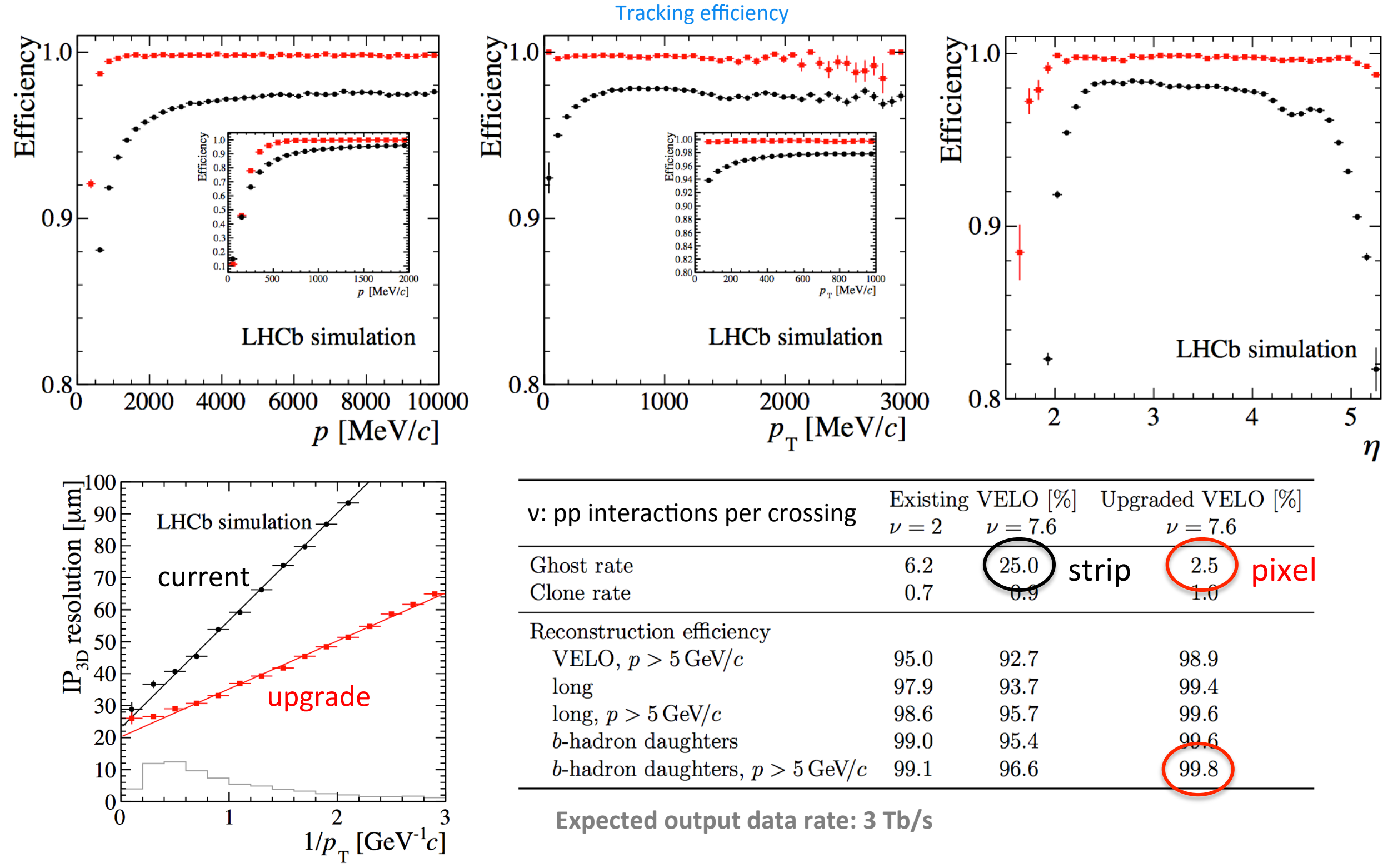

\begin{tabular}{|c|c|c|}
\hline v: pp interactions per crossing & $\begin{array}{l}\text { Existing VELO [\%] } \\
\nu=2 \quad \nu=7.6\end{array}$ & $\begin{array}{c}\text { Upgraded VELO [\%] } \\
\nu=7.6\end{array}$ \\
\hline Ghost rate & 6.2 & pixe \\
\hline Clone rate & 0.7 & \\
\hline \multicolumn{3}{|l|}{ Reconstruction efficiency } \\
\hline $\mathrm{VELO}, p>5 \mathrm{GeV} / c$ & 95.0 & 98.9 \\
\hline long & 97.9 & 99.4 \\
\hline long, $p>5 \mathrm{GeV} / c$ & 98.6 & 99.6 \\
\hline$b$-hadron daughters & 99.0 & \\
\hline$b$-hadron daughters, $p>5 \mathrm{GeV} / c$ & 99.1 & \\
\hline
\end{tabular}

Expected output data rate: $3 \mathrm{~Tb} / \mathrm{s}$

current: current technology with upgrade condition 


\section{Upstream Tracker}

- Reconstruct downstream tracks of particles decaying after the $\operatorname{VELO}\left(\mathrm{K}_{\mathrm{s}} \rightarrow \pi^{+} \pi^{-}, \wedge \rightarrow \mathrm{p} \pi\right.$, etc. $)$

- Reconstruct upstream tracks: slow momentum particles that bend out of the acceptance.

- Improve momentum resolution and signal purity of long tracks.

- $\mathbf{p}_{\mathrm{T}}$ estimate of charged tracks for fast trigger tracking

- $\sigma\left(p_{\mathrm{T}}\right) / \mathrm{p}_{\mathrm{T}} \sim 15 \%$ in the $\mathrm{p}_{\mathrm{T}}$ range of $0.5-10 \mathrm{GeV} / \mathrm{c}$.
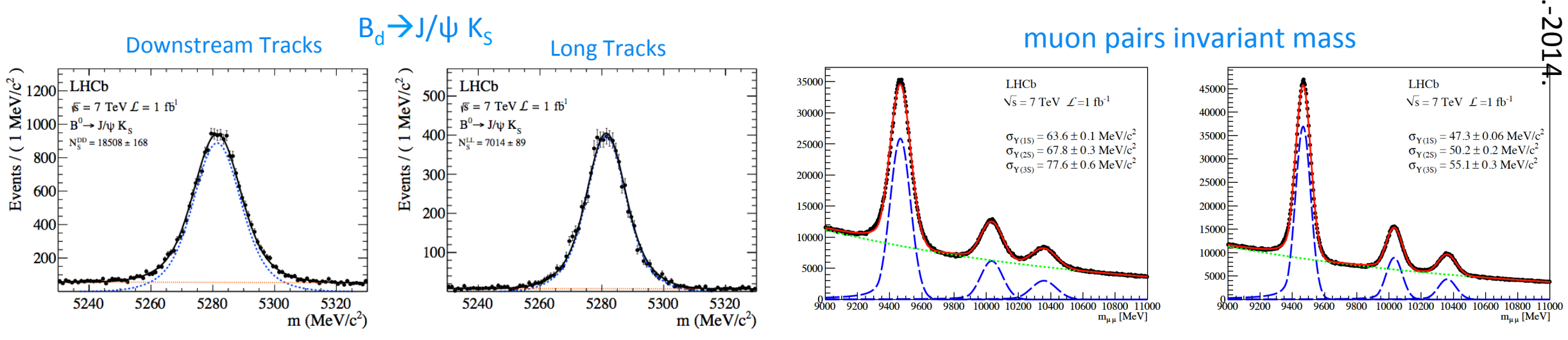

mass resolution improves by about $25 \%$ 


\section{Upstream Tracker (II)}

- Material budget kept to minimum: 1\% $\mathrm{X}_{0}$ per plane. Light mechanics and cooling system.

- $\quad$ Single-sided silicon strip sensors $250 \mu \mathrm{m}$ thick instead of $500 \mu \mathrm{m}$. Strip pitch and length adapted to the particle flux depending on the position.

- $\quad$ Single-hit resolution of $50 \mu \mathrm{m}$.

- Improved coverage by overlapping sensors.

- Closer to beam pipe to improve the small-angle acceptance.

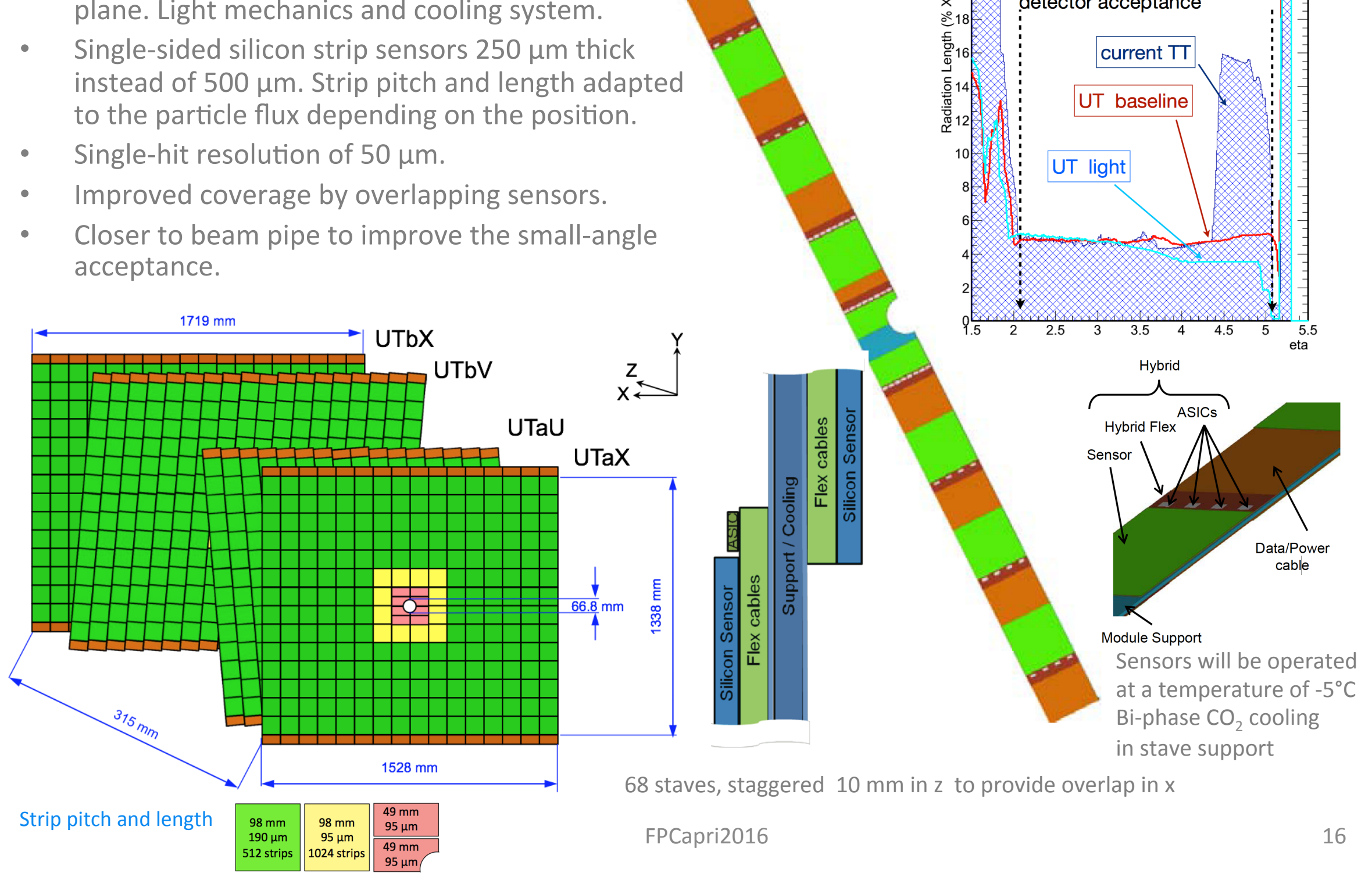

Radiation Length

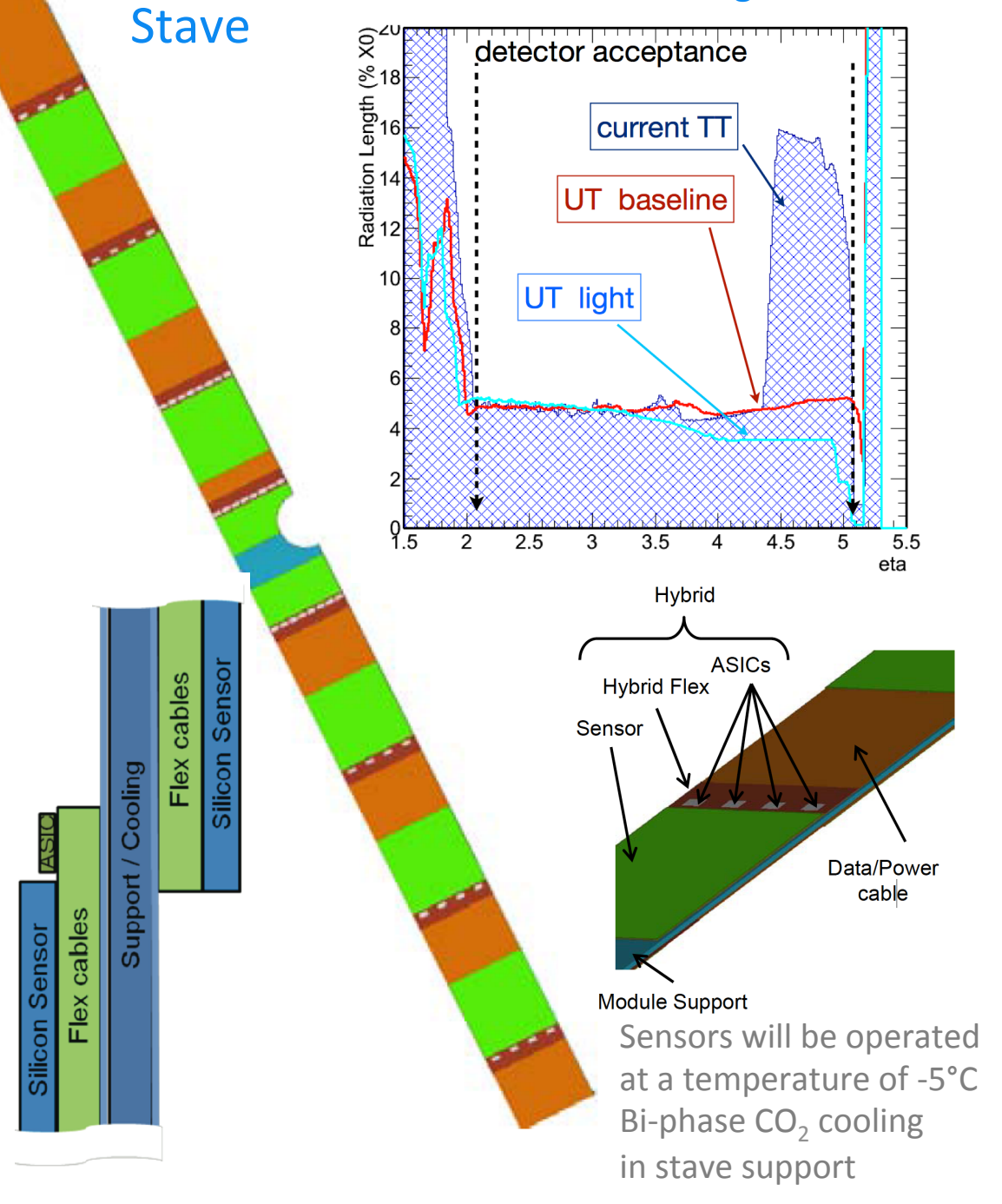

Stave

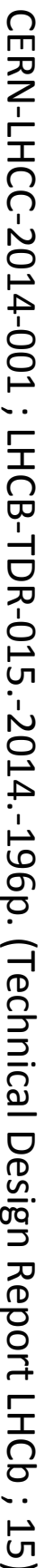

FPCapri2016 


\section{SciFi: the Downstream Tracker}

- Scintillating Fibre Trackers covering the full acceptance: $5 \times 6 \mathrm{~m}^{2}$

- A SciFi detector module is made of multiple layers of $2.5 \mathrm{~m}$ long scintillating fibres of $250 \mu \mathrm{m}$ diameter.

- Very light and uniform material distribution: $X / X_{0}=2.6 \%$ per station.

- The fibres are read by SiPM.

- The SiPMs need to be cooled to $-40^{\circ} \mathrm{C}$ to mitigate radiation damages.

- Expected 60-100 $\mu \mathrm{m}$ spatial resolution.

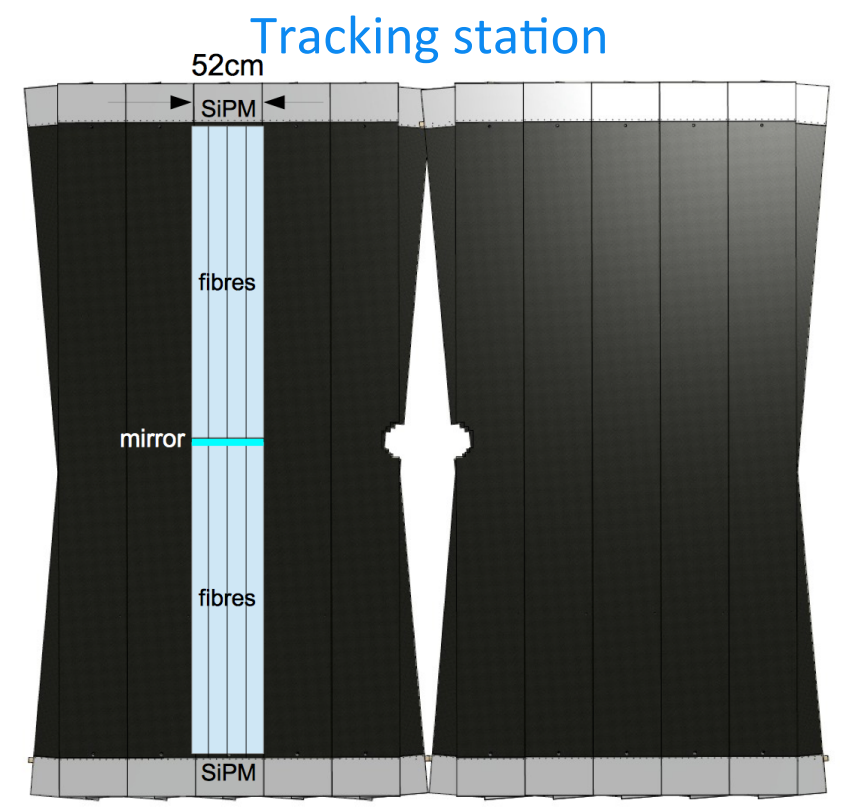

modules $x \cup \vee x$

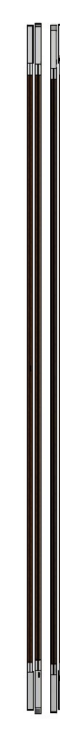

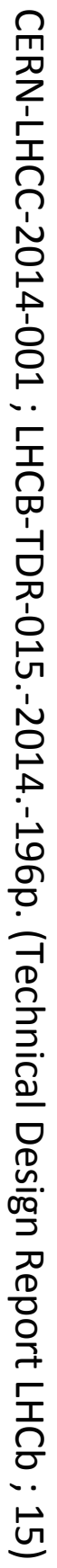
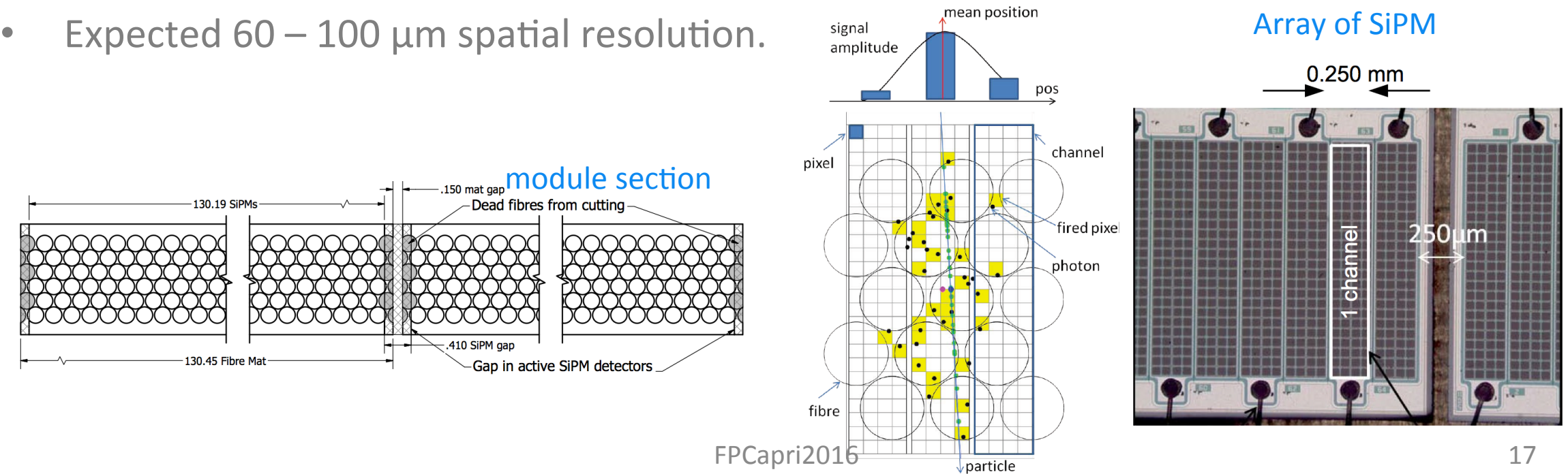

Array of SiPM 


\section{Tracking performance}
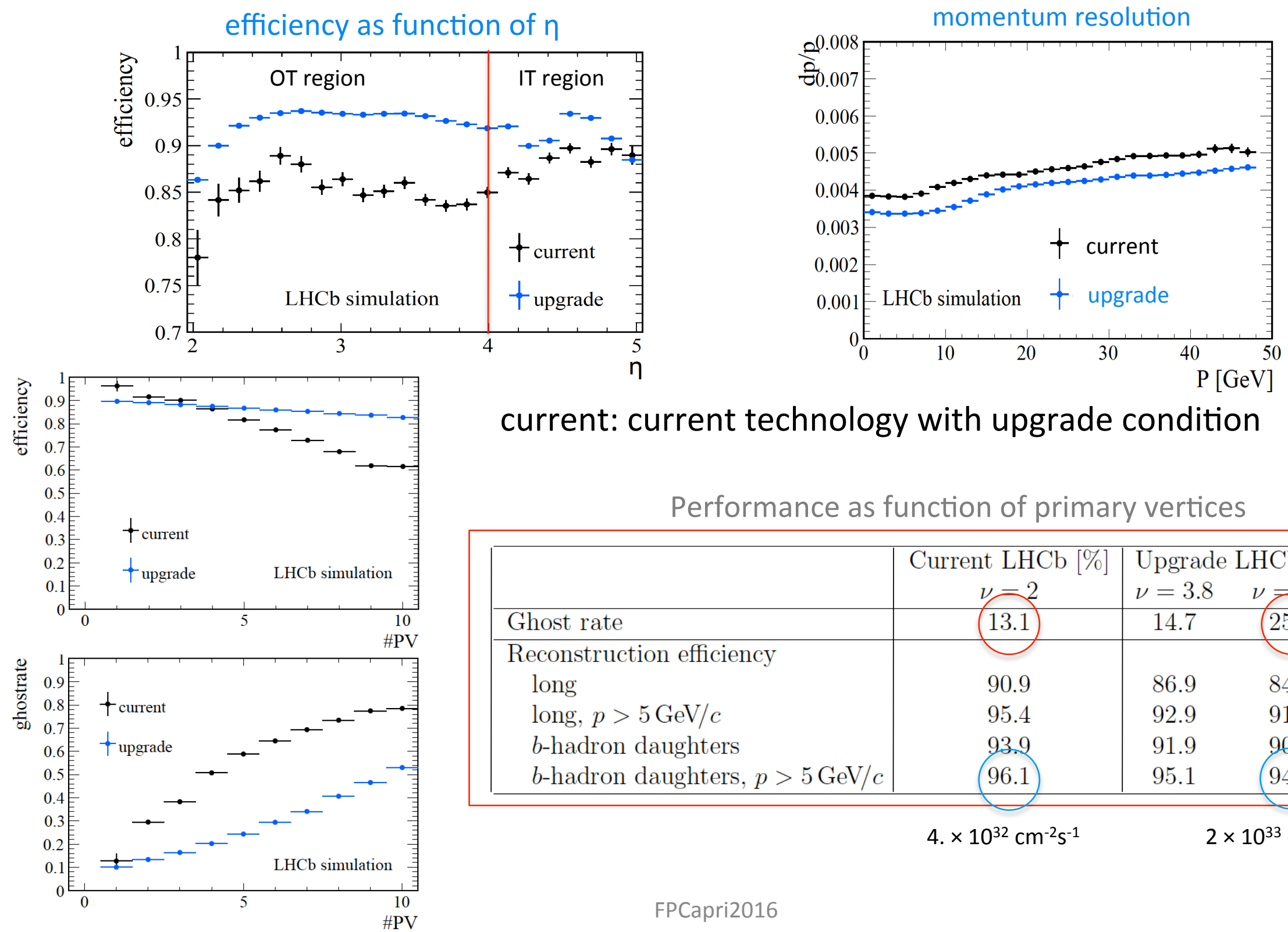

current: current technology with upgrade condition

Performance as function of primary vertices

\begin{tabular}{|l|c|cc|}
\hline & Current LHCb [\%] & Upgrade LHCb [\%] \\
& $\nu=3.8$ & $\nu=7.6$ \\
\hline Ghost rate & 13.1 & 14.7 & 25.5 \\
\hline Reconstruction efficiency & & & \\
long & 90.9 & 86.9 & 84.5 \\
long, $p>5 \mathrm{GeV} / c$ & 95.4 & 92.9 & 91.5 \\
$b$-hadron daughters & 93.9 & 91.9 & 99.6 \\
$b$-hadron daughters, $p>5 \mathrm{GeV} / c$ & 96.1 & 95.1 & 94.2 \\
\hline
\end{tabular}

4. $\times 10^{32} \mathrm{~cm}^{-2} \mathrm{~s}^{-1}$ 


\section{$\mathrm{RICH}$ detector}

- The overall structure of RICH-1 and $\mathrm{RICH}-2$ unchanged.

- HPD photon detectors will be replaced with MaPMT

- 1920 MaPMT in RICH-1 and 2560 in $\mathrm{RICH} 2$

- The optical layout of RICH 1 has to be modified to reduce the hit occupancy.

- Increasing the focal length of the spherical mirrors halving the occupancy.

- Remove the Aerogel radiator in RICH-1.

- $\sim 3.5 \%$ of $X_{0}$

- The $\mathrm{K}$ and $\pi$ threshold in $\mathrm{C}_{4} \mathrm{~F}_{10}$ are $9.3 \mathrm{GeV} / \mathrm{c}$ and $2.6 \mathrm{GeV} / \mathrm{c}$.
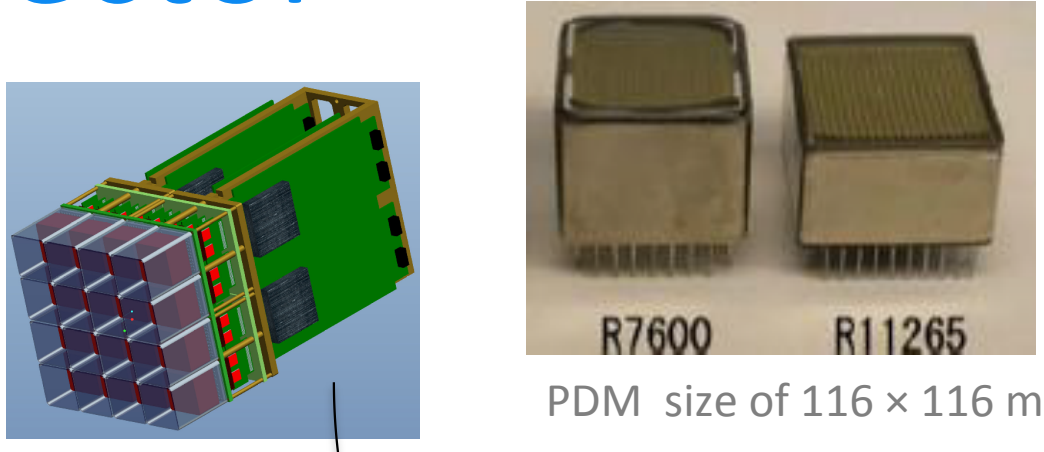

PDM size of $116 \times 116 \mathrm{~mm}^{2}$

R11265 MaPMT from Hamamatsu.

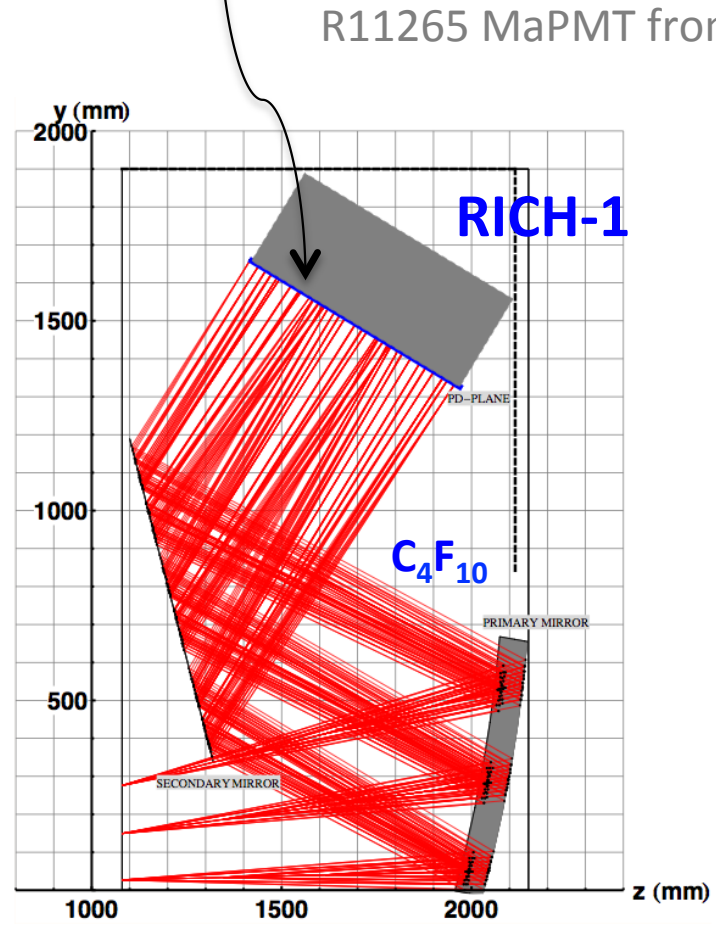




\section{RICH PID performance}

\section{Running the full simulation and reconstruction chain in $B_{s} \rightarrow \phi \phi$}

\section{Compare upgraded with current geometry}
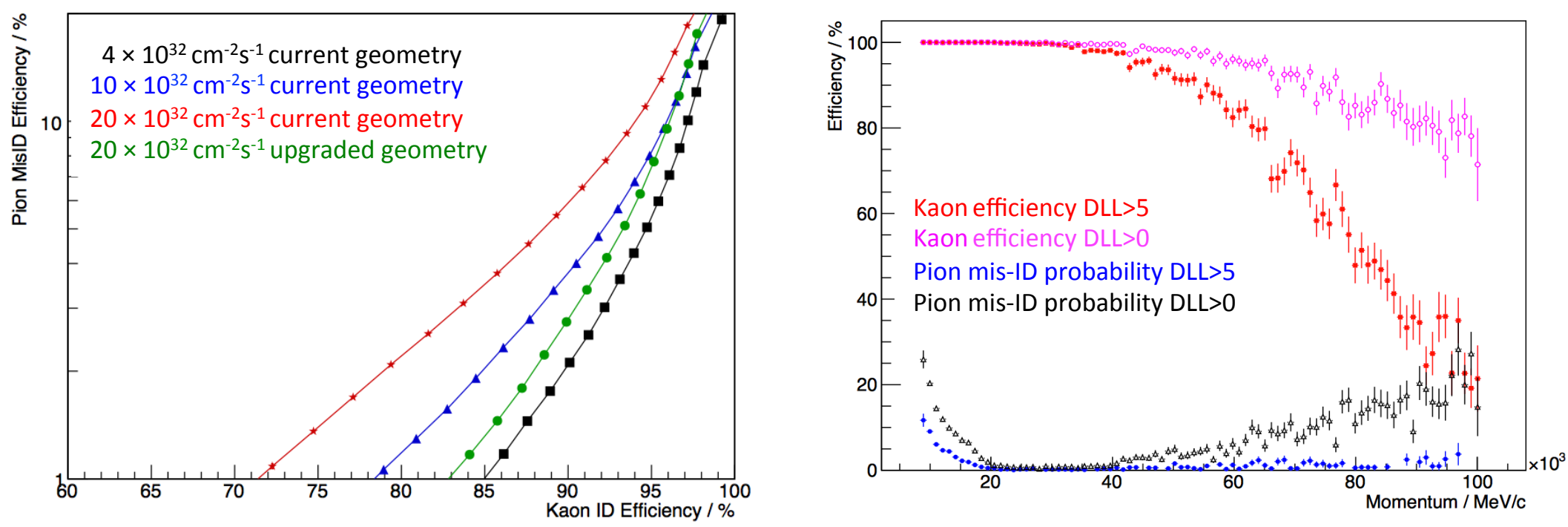


\section{MUON system}

M1

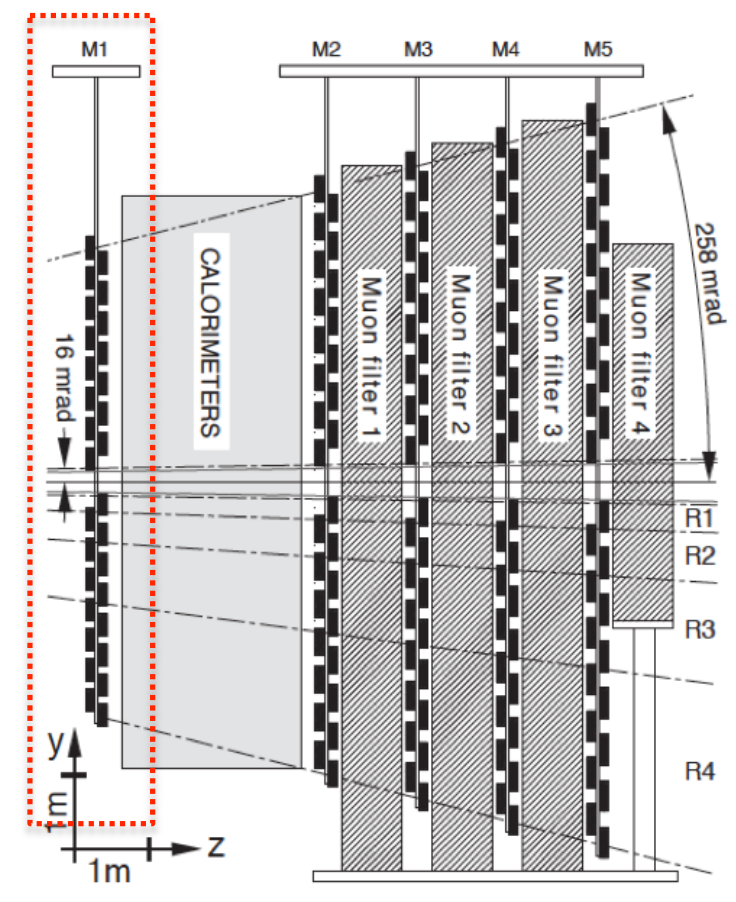

Anode-pad triple-GEM detectors for the R1 regions, MWPCs for the external regions.

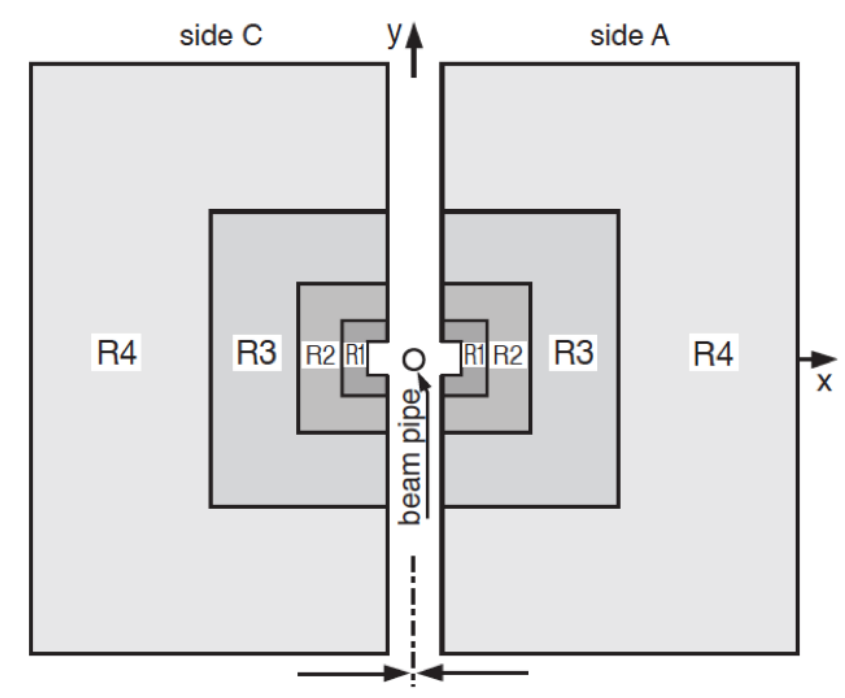

- M1 will be removed: currently used by the L0 trigger.

- High particle flux in the innermost regions of station M2.

Shielding will be installed around the beam-pipe, behind the HCAL, to reduce the occupancy in these regions. 


\section{MUON ID Performance}
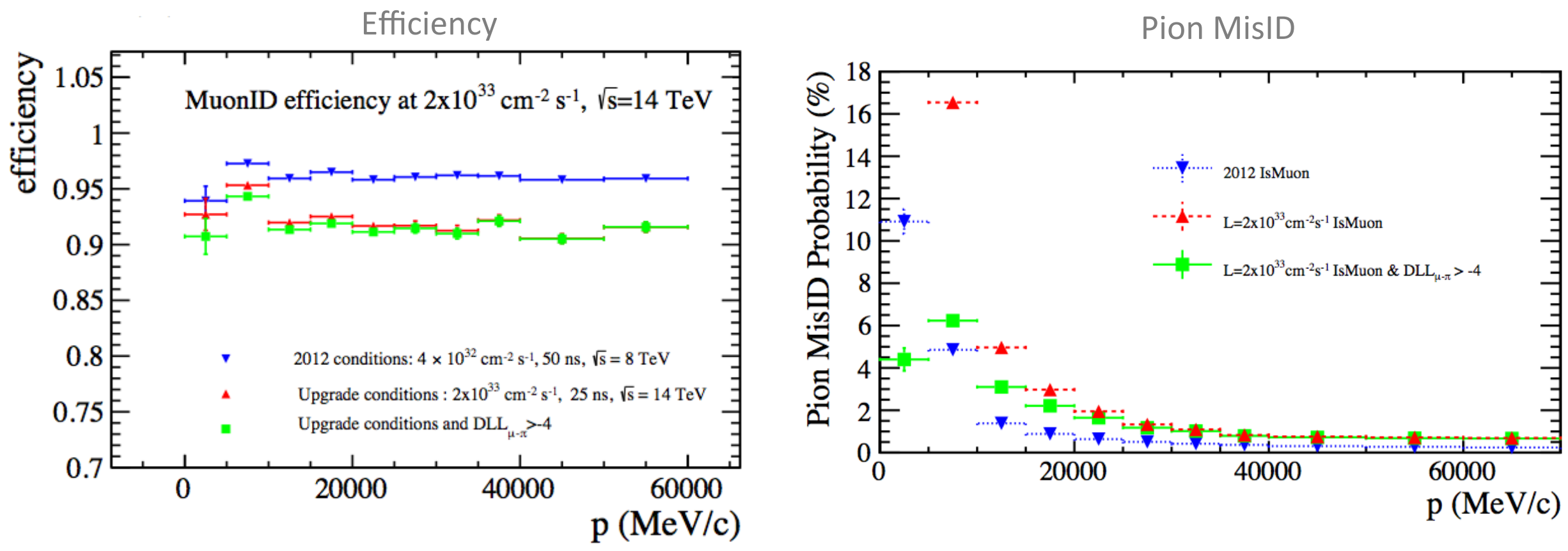

- The upgrade and current performance are obtained from a simulated sample of $B_{s} \rightarrow \mu^{+} \mu^{-}$events and are evaluated for single muon.

- $\mathrm{DLL}_{\mu \pi}$ variable: is based on the distance of matching hits from the extrapolated track in the muon stations, combined with the information coming from $\mathrm{RICH}$ and calorimeter detectors. 


\section{Calorimeters}

- The scintillating pad detector (SPD) and the pre-shower (PS) will be removed.

- Very little effect for the higher electron momentum: $p>10 \mathrm{GeV} / \mathrm{c}$

- A reduction of 10 to $15 \%$ in the efficiency is expected at a fixed background retention for lower momenta.

\begin{tabular}{|c|c|c|}
\hline $\begin{array}{c}\text { Momentum } \\
(\mathrm{GeV} / \mathrm{c})\end{array}$ & $\begin{array}{c}\text { SPD/PS } \\
\nu=7.6\end{array}$ & $\begin{array}{c}\text { no SPD/PS } \\
\nu=7.6\end{array}$ \\
\hline \multicolumn{3}{|c|}{ Selection efficiency $80 \%$} \\
\hline $0<p<10$ & 3.2 & 9.0 \\
\hline$p>10$ & 0.29 & 0.32 \\
\hline \multicolumn{3}{|c|}{ Selection efficiency $90 \%$} \\
\hline $0<p<10$ & 12 & 18 \\
\hline$p>10$ & 1.3 & 1.4 \\
\hline
\end{tabular}
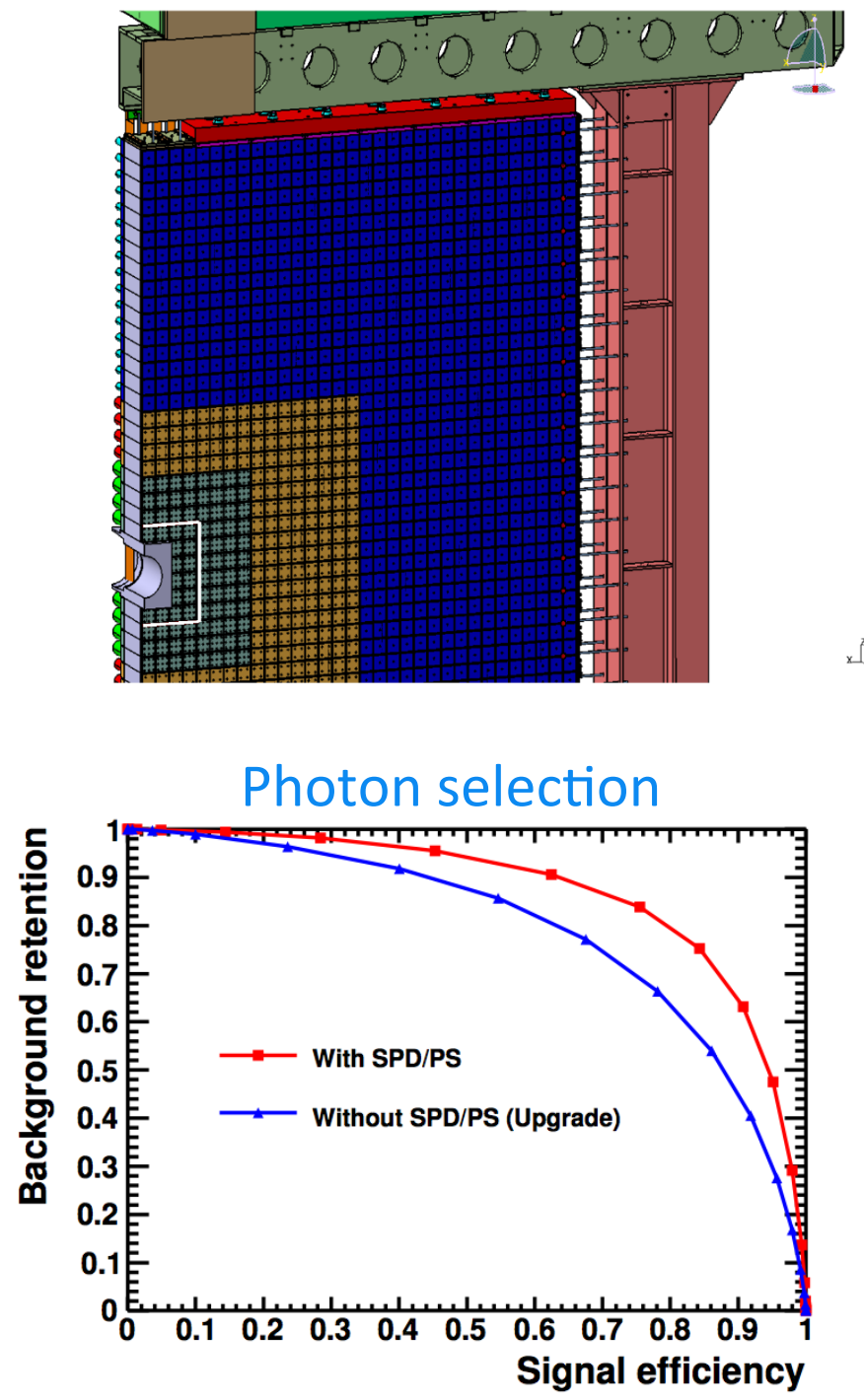


\section{Upgrade milestone evolution}

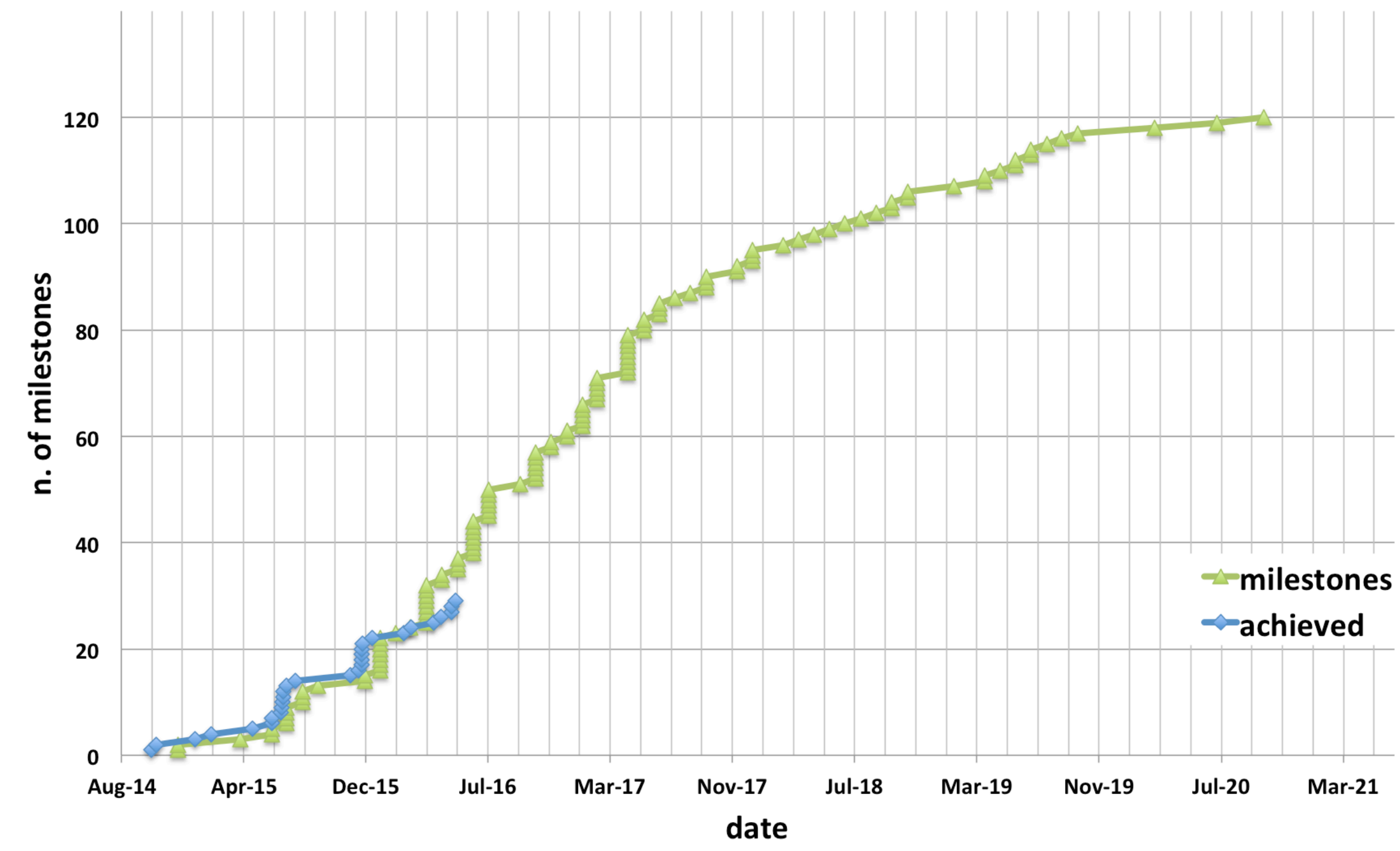




\section{LHCb upgrade beyond 2023}

- Upgrade phase Ib: Run 4, $2027-2030$

- Run still at 2. $\times 10^{33} \mathrm{~cm}^{-2} \mathrm{~s}^{-1}$, with an improved detector.

- Upgrade phase II: Run 5, beyond 2031

- Run at 2. $\times 10^{34} \mathrm{~cm}^{-2} \mathrm{~s}^{-1}, \mathrm{v}^{\sim} 40$ to collect $\sim 300 \mathrm{fb}^{-1}$

- Implies a radical change of the detector.

- 4D tracking with timing pixel

- "Beyond the LHCb phase 1 upgrade" Theater of Dreams, Manchester 6-7 April 2016

$\begin{array}{cl}\text { Phenomenological } & \begin{array}{l}\text { Physics benchmarks beyond LS4 } \\ \text { Bragg Lecture Theatre, Schuster Laboratory, University of Manchester }\end{array} \\ \text { Theoretical Contributions } & \text { Searches for NP in CP violation } \\ \text { Bragg Lecture Theatre, Schuster Laboratory, University of Manchester } \\ \text { Searches for NP in rare decays } \\ \text { Bragg Lecture Theatre, Schuster Laboratory, University of Manchester }\end{array}$




\section{Sensitivity prospects}

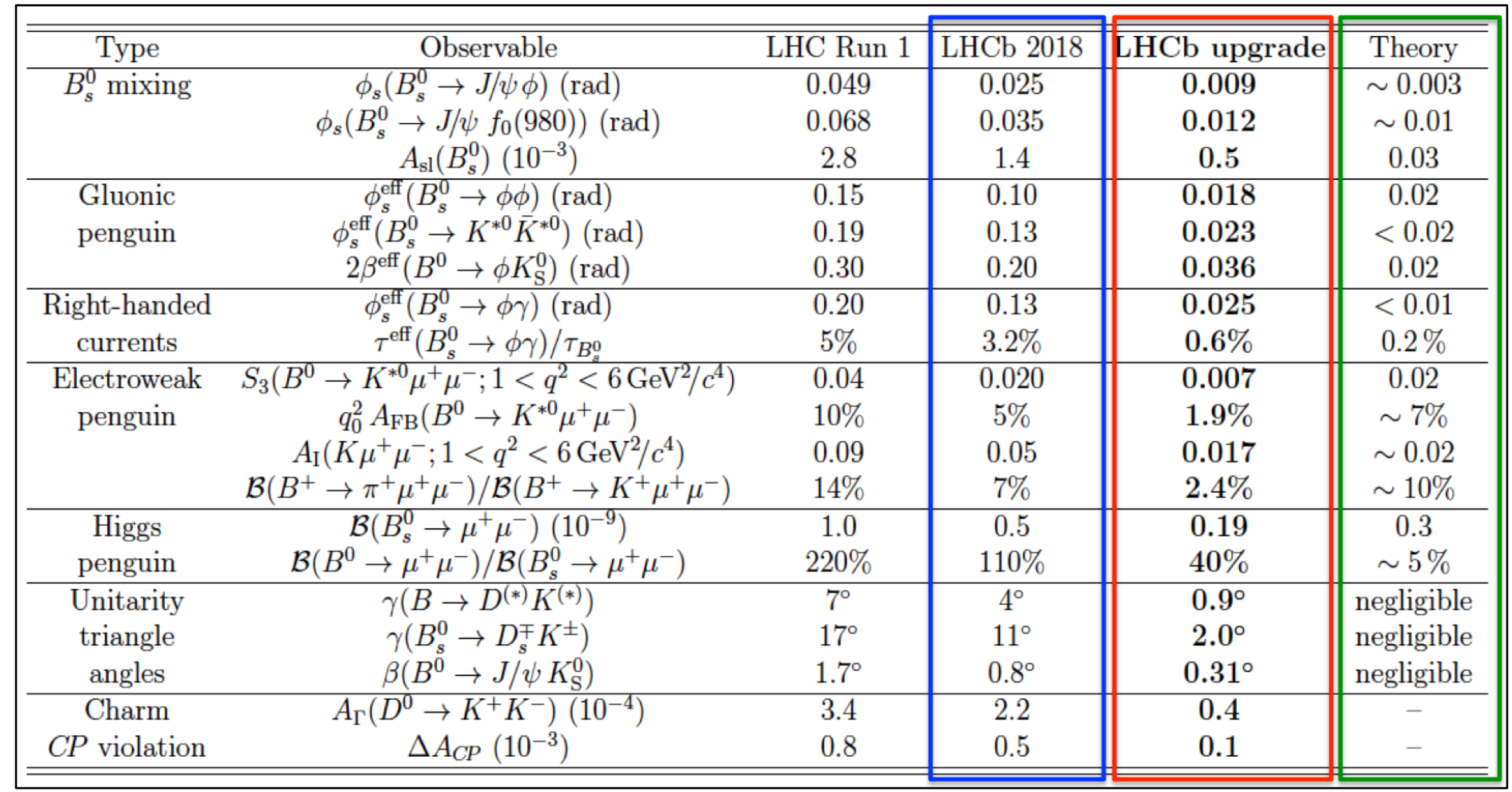

- Before the upgrade $\left(8 \mathrm{fb}^{-1}\right)$

- After the upgrade $\left(50 \mathrm{fb}^{-1}\right)$

- Theory uncertainty (as far as we know today)
The extrapolations assume:

- Precisions scale as VL.

- Gain $\times 2$ on fully hadronic decays

- HLT and analysis performance as in Run I

- Backgrounds as in Run I. 


\section{$\gamma$ from trees}

- Combining several independent decay modes is the key to achieve the ultimate precision.

\begin{tabular}{|l|l|l|}
\hline LHCb measurement & Type/ Dataset & Reference \\
\hline $\mathrm{B}^{+} \rightarrow \mathrm{DK}^{+} \mathrm{D} \rightarrow 2 \mathrm{~h}, 4 \mathrm{~h}$ & ADS/(q-)GLW $\left(3 \mathrm{fb}^{-1}\right)$ & arXiv:1603.08993 \\
\hline $\mathrm{B}^{0} \rightarrow \mathrm{DK} \pi$ & Dalitz $\left(3 \mathrm{fb}^{-1}\right)$ & arXiv: 1602.03455 \\
\hline $\mathrm{B}^{0} \rightarrow \mathrm{DK}^{*} \mathrm{D} \rightarrow \mathrm{Ks} \pi \tau$ & GGSZ MD $\left(3 \mathrm{fb}^{-1}\right)$ & arXiv: 1605.01082 \\
\hline $\mathrm{B}^{+} \rightarrow \mathrm{DK}^{+} \mathrm{D} \rightarrow \mathrm{hh} \pi^{0}$ & ADS/q-GLW $\left(3 \mathrm{fb}^{-1}\right)$ & PRD 91(2015) 112014 \\
\hline $\mathrm{B}^{+} \rightarrow \mathrm{DK} \pi \pi, \mathrm{D} \rightarrow 2 \mathrm{~h}$ & ADS/GLW $\left(3 \mathrm{fb}^{-1}\right)$ & PRD 92 (2015) 112005 \\
\hline $\mathrm{B}^{0} \rightarrow \mathrm{DK}^{*} \mathrm{D} \rightarrow 2 \mathrm{~h}$ & ADS $\left(3 \mathrm{fb}^{-1}\right)$ & PRD $90(2014) 112002$ \\
\hline $\mathrm{B}^{+} \rightarrow \mathrm{DK} \mathrm{D} \rightarrow \mathrm{K}_{\mathrm{s}} \mathrm{hh}$ & GGSZ MI $\left(3 \mathrm{fb}^{-1}\right)$ & JHEP $10(2014) 097$ \\
\hline $\mathrm{B}^{+} \rightarrow \mathrm{DK}, \mathrm{D} \rightarrow \mathrm{KsK} \pi$ & ADS $\left(3 \mathrm{fb}^{-1}\right)$ & PLB 733 (2014) 36 \\
\hline $\mathrm{B}_{\mathrm{s}} \rightarrow \mathrm{D}_{\mathrm{s}} \mathrm{K}, \mathrm{D}_{\mathrm{s}} \rightarrow \mathrm{hhh}$ & Time dep $\left(1 \mathrm{fb}^{-1}\right)$ & JHEP 11 (2014) 060 \\
\hline
\end{tabular}

LHCb combined result

$$
\text { LHCb: } \quad \gamma=\left(70.9_{-8.5}^{+7.1}\right)^{\circ}
$$

BaBar : $\quad \gamma=\left(69_{-16}^{+17}\right)^{\circ}$ PRD 87 (2013) 052015

Belle: $\quad \gamma=\left(73_{-14}^{+15}\right)^{\circ} \quad$ arXiv:1301.2033

$\sigma(\gamma) \approx 4^{\circ}$ by 2018 and sub-degree precision by the end of the experimental programme

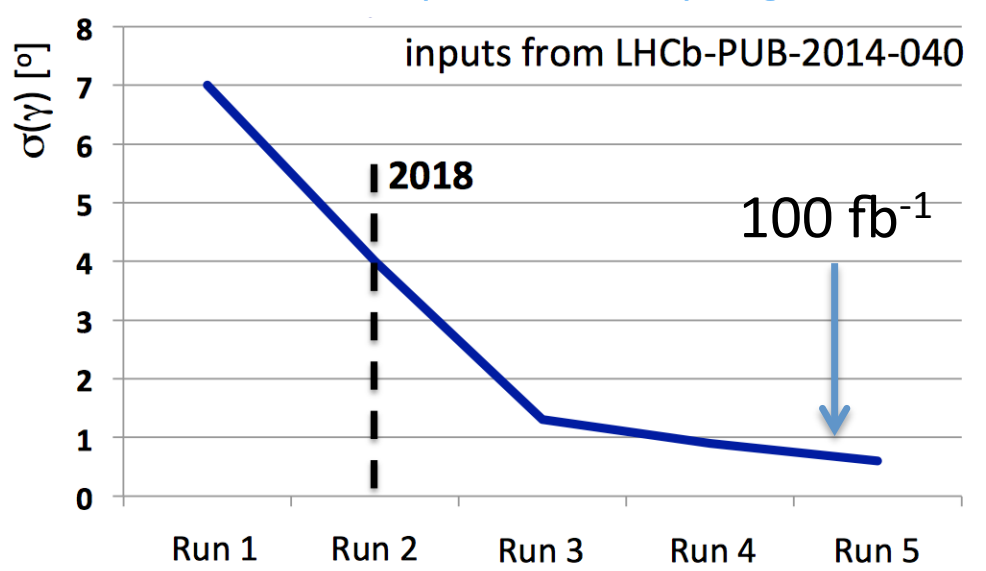

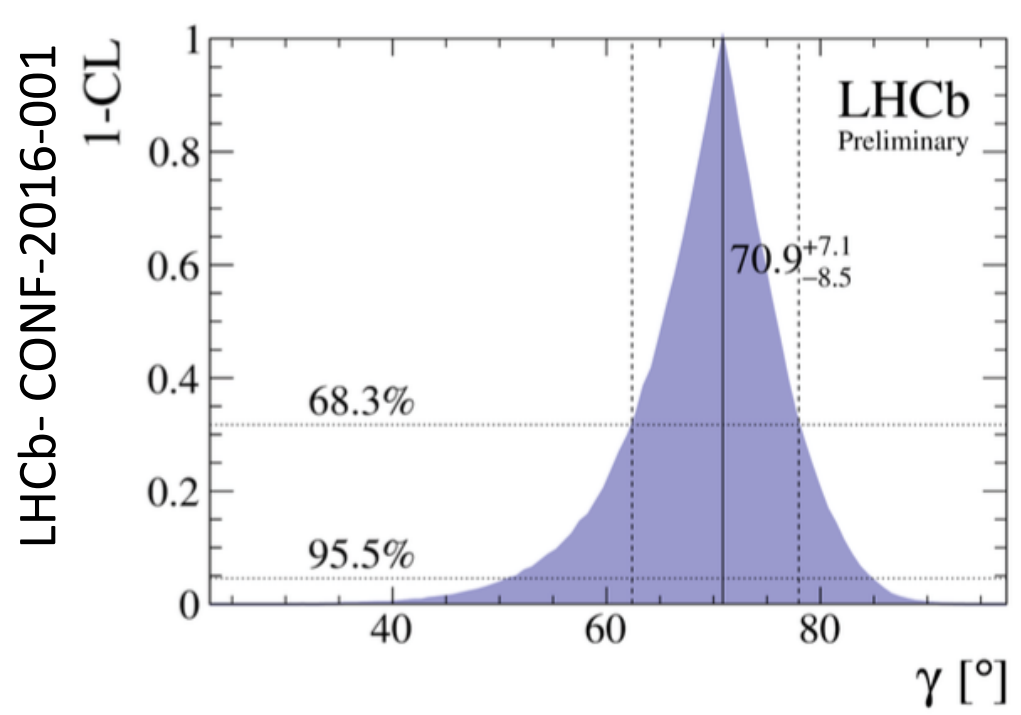

FPCapri2016 


\section{$\mathrm{CP}$ violation induced by $\mathrm{B}_{\mathrm{s}}$ mixing: $\phi_{\mathrm{s}}$}

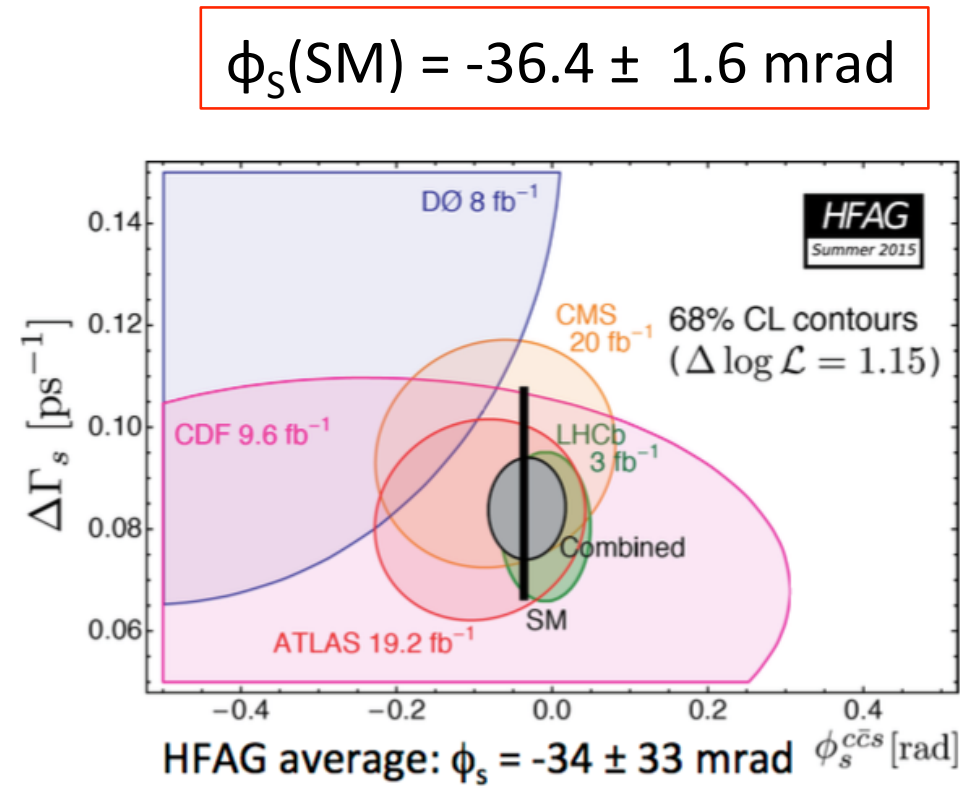

- $\quad$ LHCb results with $3 \mathrm{fb}^{-1}$

- $\mathbf{B}_{\mathrm{s}} \rightarrow \mathbf{J} / \boldsymbol{\Psi K}^{+} \boldsymbol{K}^{-}, \phi_{\mathrm{s}}=-58 \pm 49 \pm 6 \mathrm{mrad}$

- Phys. Rev. Lett. 114 (2015) 041801

- $\mathbf{B}_{\mathrm{s}} \rightarrow \mathrm{J} / 4 \boldsymbol{\pi}^{+} \boldsymbol{\pi}^{-}, \phi_{\mathrm{s}}=70 \pm 68 \pm 8 \mathrm{mrad}$

- Phys. Lett. B736 (2014) 186

- $\mathrm{B}_{\mathrm{s}} \rightarrow \mathrm{D}^{+}{ }_{\mathrm{s}} \mathrm{D}_{\mathrm{s}}^{-}=20 \pm 170 \pm 20 \mathrm{mrad}$

- Phys. Rev. Lett. 113 (2014) 211801

LHCb average: $\phi_{\mathrm{s}}=-10 \pm 40 \mathrm{mrad}$

We don't envisage systematic uncertainties to become limiting factors
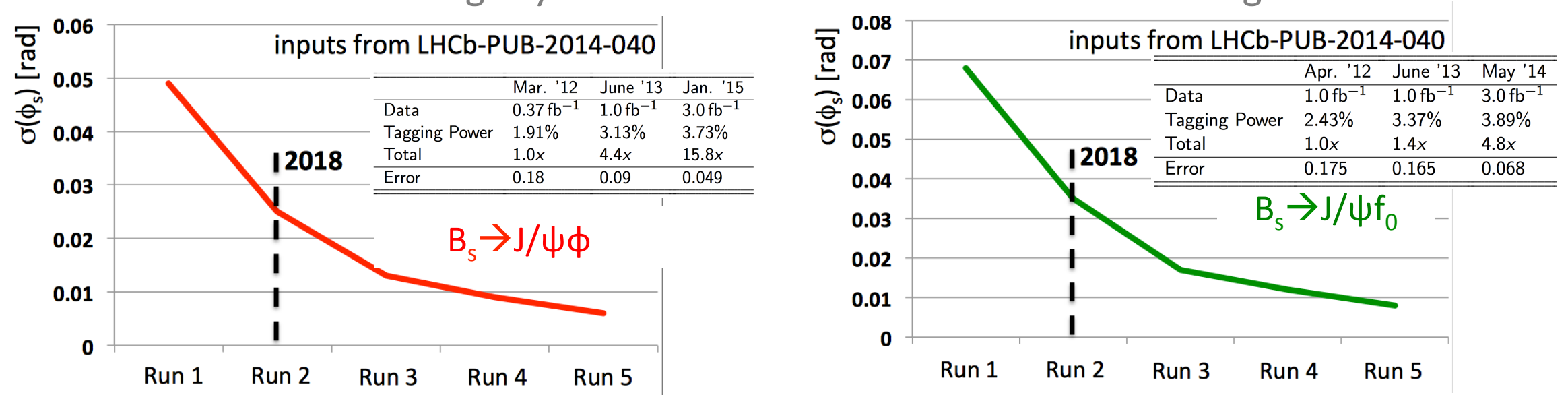

$\sigma\left(\phi_{S}\right) \sim 4$ mrad with $300 \mathrm{fb}^{-1} \quad$ FPCapri2016

$\sigma\left(\phi_{\mathrm{S}}\right) \sim 5$ mrad with $300 \mathrm{fb}^{-1} \quad 28$ 


\section{$C P$ violation in $B_{s} \rightarrow \phi \phi$}

Interference between $\mathrm{B}_{\mathrm{S}}-\underline{B}_{\mathrm{S}}$ mixing and the loop-induced decay amplitude.

- FCNC gluonic $b \rightarrow$ sss penguin

- Provides an excellent probe of new heavy particles entering the penguin quantum loops.

- $\quad$ LHCb result with full Run 1 data set $\left(3 \mathrm{fb}^{-1}\right)$, with approximately 4000 events:

$$
\phi_{s}=(-170 \pm 150 \pm 30) \mathrm{mrad}
$$

- Overall precision comparable to golden $b \rightarrow c$ cs modes.

- No sign of discrepancy.
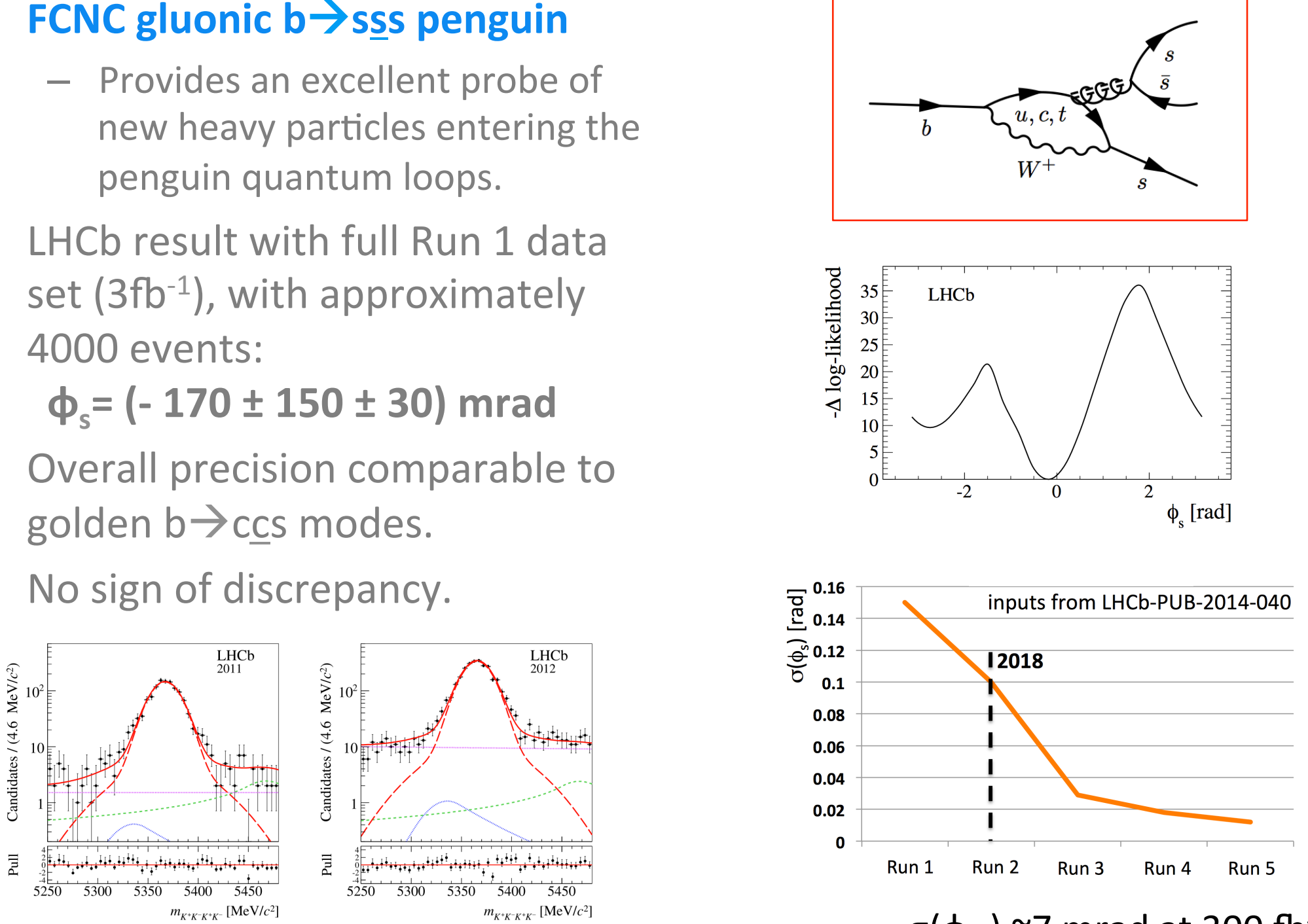
CMS \& LHCb
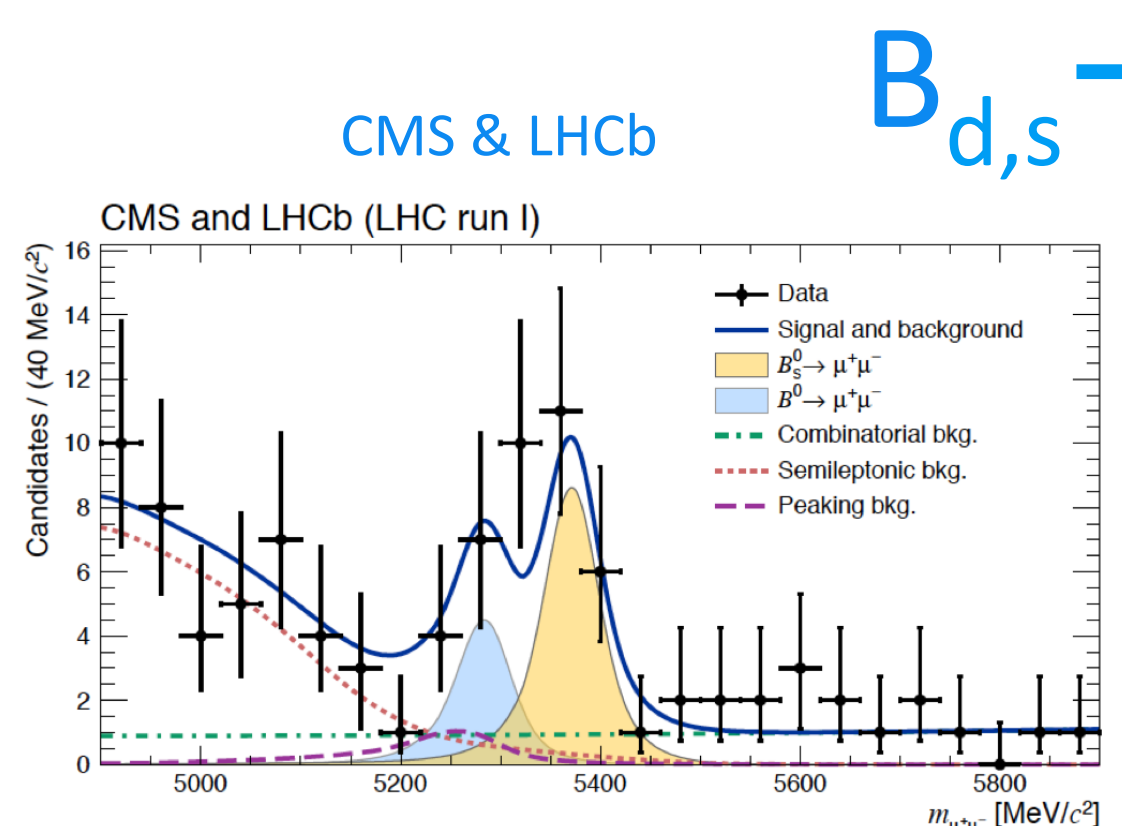

$$
\begin{aligned}
\mathcal{B}\left(B_{s} \rightarrow \mu^{+} \mu^{-}\right) & =2.8_{-0.6}^{+0.7} \times 10^{-9} \\
\mathcal{B}\left(B^{0} \rightarrow \mu^{+} \mu^{-}\right) & =3.9_{-1.4}^{+1.6} \times 10^{-10}
\end{aligned}
$$

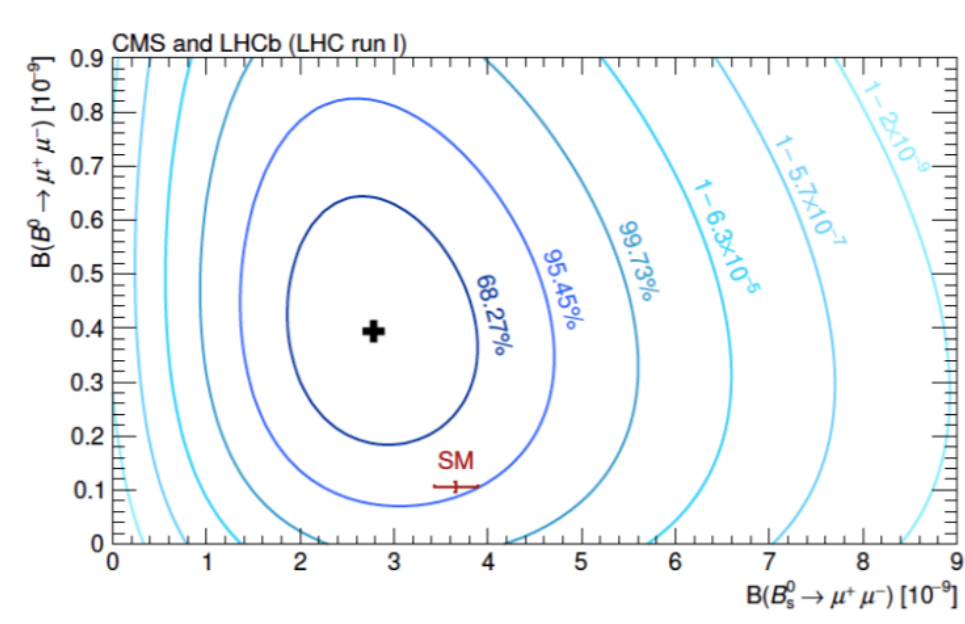

FPCapri2016

FPCapri2016
Significance of $B_{s} \rightarrow \mu \mu$ at $6.2 \sigma$

- First observation

- Compatibility with the SM at $1.2 \sigma$

$B^{0} \rightarrow \mu \mu$ hypothesis:

- Excess of events at the $3 \sigma$ level observed with respect to background.

- Compatible with SM at $2.2 \sigma$

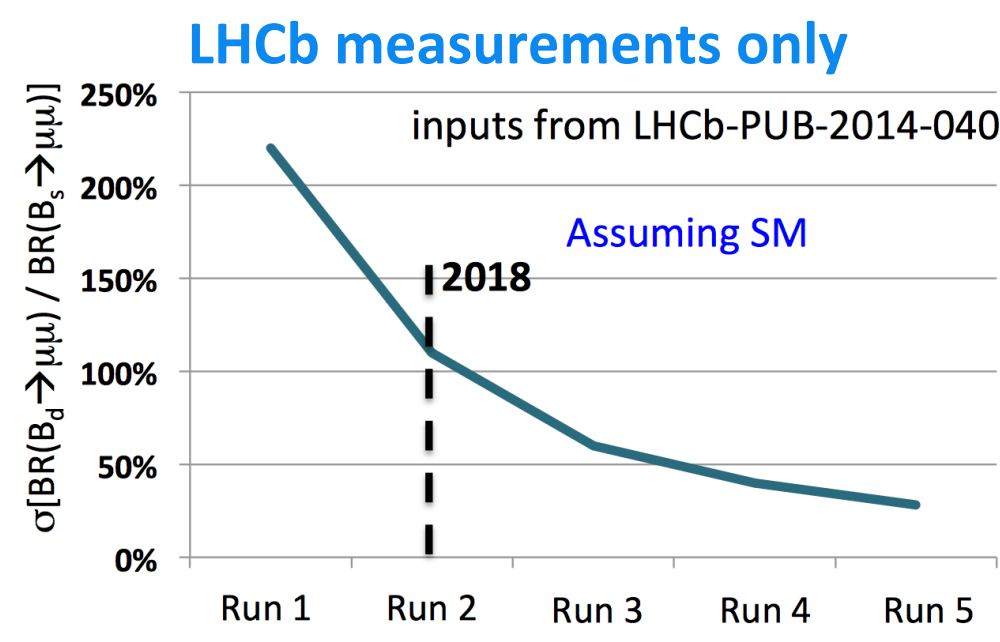

By the end of Run 4 measurements will still be dominated by experimental uncertainty. Uncertainty $<10 \%$ on the ratio $B R(B d) / B R(B s)$ is at reach with $300 \mathrm{fb}^{-1}$

New observables: effective lifetime,... 


\section{Conclusions}

- LHCb has performed very well in Run 1

Confirming to date the Standard Model predictions.

- Improvements of $\mathrm{LHCb}$ results are expected in Run 2

Additional 5-6 $\mathrm{fb}^{-1}$ expected by 2018 , to be collected with the present detector, with improved trigger capabilities.

- The LHCb upgraded detector shall start taking data in 2020

New PCle based read out and full HLT software trigger at $40 \mathrm{MHz}$.

Optimized tracking system and still good PID.

The preparation of the upgrade is progressing well.

- LHCb prospects look excellent

Heavy flavour physics has still large room for improvements.

Key measurements are far from being limited by systematic uncertainties. $\mathrm{LHCb}$ has great potential of discovering indirect evidence of NP in future measurements. 
The End 


\section{Present $1 \mathrm{MHz}$ DAQ system \\ Push-protocol with centralized flow-control}

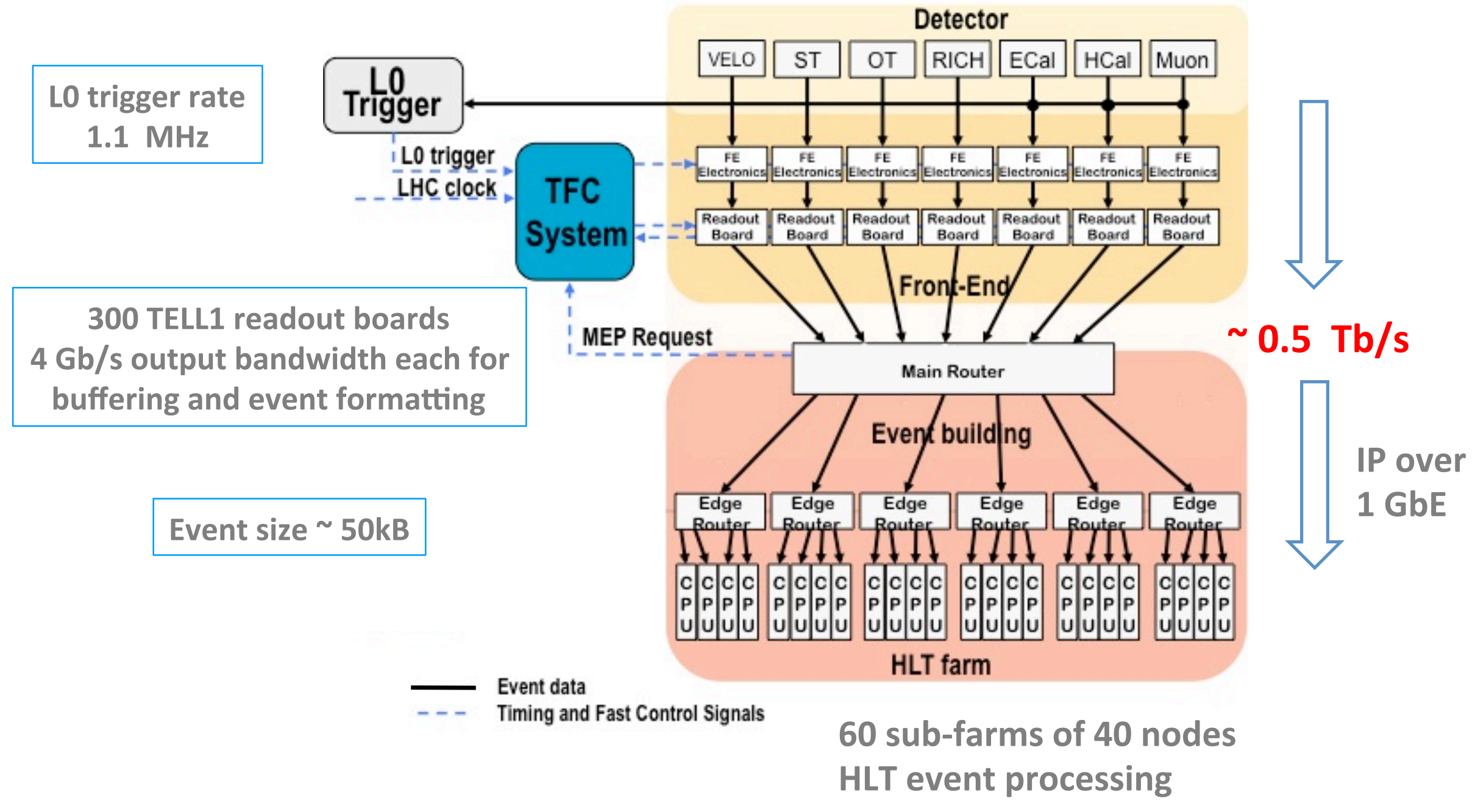




\section{Trigger in Run1}

- LO Trigger: $\mathbf{4 0} \mathrm{MHz} \rightarrow \mathbf{1 M H z}$

$40 \mathrm{MHz}$ bunch crossing rate
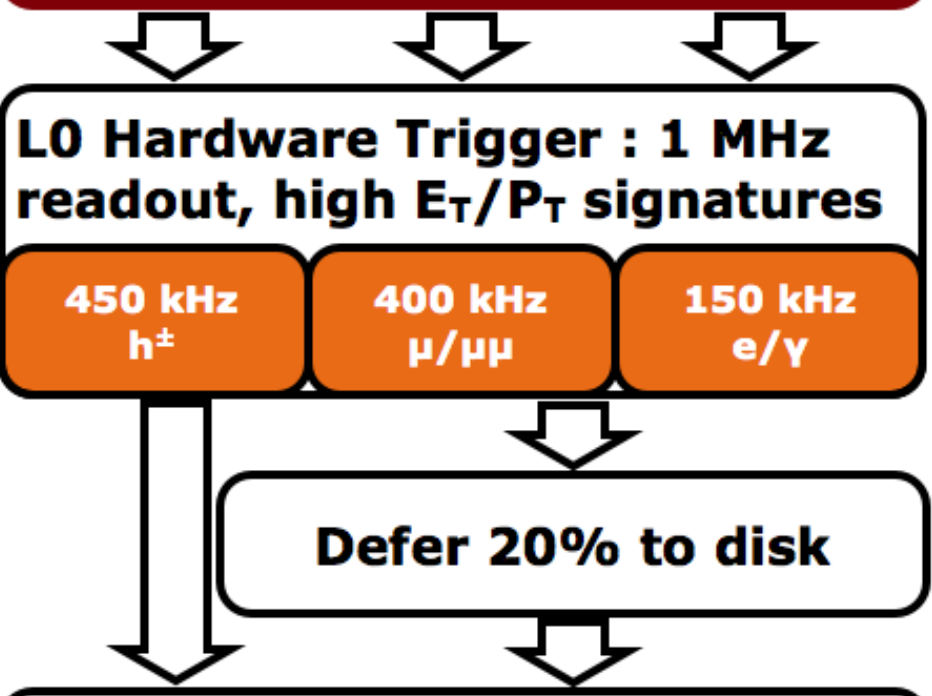

Software High Level Trigger 29000 Logical CPU cores

Offline reconstruction tuned to trigger time constraints

Mixture of exclusive and inclusive selection algorithms

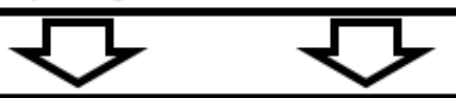

$5 \mathrm{kHz}$ Rate to storage
- ECAL, HCAL and MUON detectors read out at $40 \mathrm{MHz}$

- $20 \%$ to disk: deferred processing

- HLT: $1 \mathrm{MHz} \rightarrow 5 \mathrm{kHz}$

- Reconstruct VELO tracks and primary vertices

HLT1 - Select events with at least one track matching $p, p_{T}$, impact parameter and track quality cuts.

- At around $100 \mathrm{kHz}$ performs inclusive or exclusive selections of the events.

HLT2

- Full track reconstruction, without particle-identification.

- Total accept rate to disk for offline analysis is around $5 \mathbf{~ k H z}$. 


\section{Trigger evolution in Run2}

- LHC stable beams during $30 \%$ of the running period: $70 \%$ of the time the CPU of the HLT farm would be idle.

- Real-time HLT1

HLT1 selects events that are temporarily stored on 5.2 PB of the farm servers.

- Buffer 10 days of continuous data taking.

- Staging after the HLT1 filter occurs at a rate of about $100 \mathrm{kHz}$ instead of $1 \mathrm{MHz}$.

- HLT1 time budget $~ 50 ~ m s$

- Deferred HLT2

Performs the final event filtering, relying on up-to-date calibration constants, with offline quality.

- HLT2 time budget: 800 ms

- Trigger algorithm: 350 trigger lines

- Output rate $\sim 12.5 \mathrm{kHz}$.

- $2.5 \mathrm{kHz}$ processed as Turbo Stream (no more raw data recorded)

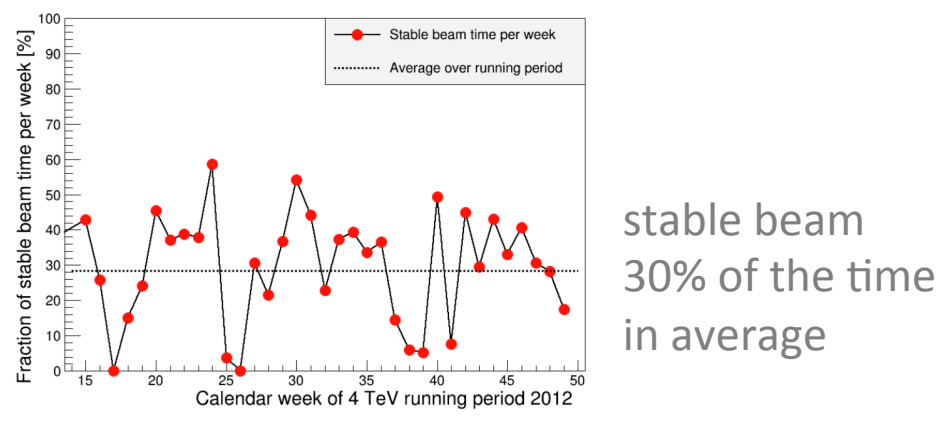

LHCb 2015 Trigger Diagram

$40 \mathrm{MHz}$ bunch crossing rate

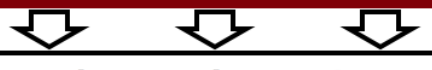

LO Hardware Trigger : 1 MHz readout, high $E_{\mathrm{T}} / \mathrm{P}_{\mathrm{T}}$ signatures

\begin{tabular}{|lll}
$450 \mathrm{kHz}$ & $400 \mathrm{kHz}$ & $150 \mathrm{kHz}$ \\
\hline
\end{tabular}

$h^{ \pm}$

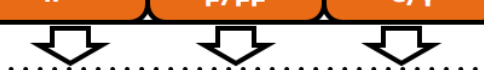
Software High Levei Trigger

Partial event reconstruction, select
displaced tracks/vertices and dimuons displaced tracks/vertices and dimuons

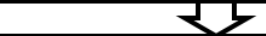

Buffer events to disk, perform online detector calibration and alignment ए人

Full offline-like event selection, mixture

of inclusive and exclusive triggers
ofine

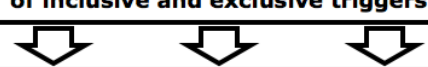

$12.5 \mathrm{kHz}$ Rate to storage 


\section{Upgrade phase Ib}

- Spectroscopy and CP-violation studies are increasingly focused on high multiplicity final states

- Extend the tracking acceptance of the tracking stations by instrumenting the internal sides of the magnet, and possibly outside the magnet.

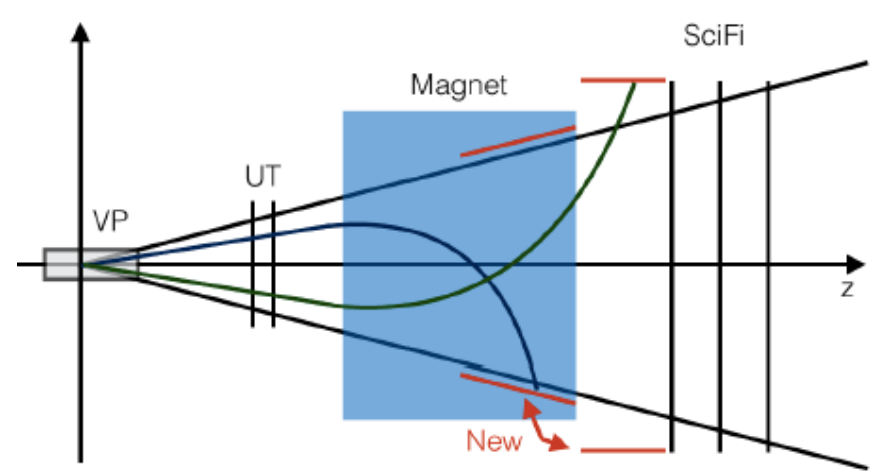

- Improve RICH performance: replace part of the MaPMT with SiPM to increase the granularity to $1 \mathrm{~mm}$ from the present $3 \mathrm{~mm}$.

- Performance with $\pi^{0}$ and $\gamma$ are still far behind analyses with charged tracks

- $B \rightarrow D K \rightarrow\left(K \pi \pi^{0}\right)_{D} K$

- Neutral reconstruction will be even worse at $2 \times 10^{33} \mathrm{~cm}^{-2} \mathrm{~s}^{-1}$ : Baseline is to replace the innermost part of ECAL, above $20 \mathrm{fb}^{-1}$ Think to different ECAL technology?

- Scintillator-W based ECAL

- CALICE-type ECAL 